\title{
Modular Three-Component Synthesis of 4-Aminoquinolines via an Imidoylative Sonogashira/Cyclization Cascade
}

\author{
Jurriën W. Collet, ${ }^{\dagger}$ Kelly Ackermans, ${ }^{\dagger}$ Jeffrey Lambregts, $^{\dagger}$ Bert U.W. Maes, ${ }^{\ddagger}$ Romano V.A. Orru, ${ }^{*}{ }^{\dagger}$ \\ and Eelco Ruijter* ${ }^{\dagger}+0$
}

${ }^{\dagger}$ Department of Chemistry and Pharmaceutical Sciences and Amsterdam Institute for Molecules, Medicines \& Systems (AIMMS), Vrije Universiteit Amsterdam, De Boelelaan 1108, $1081 \mathrm{HZ}$ Amsterdam, The Netherlands

¥Organic Synthesis, Department of Chemistry, University of Antwerp, Groenenborgerlaan 171, 2020 Antwerp, Belgium

\section{Supporting Information}

ABSTRACT: We developed a one-pot, two-stage synthetic route to substituted 4-aminoquinolines involving an imidoylative Sonogashira coupling followed by acid-mediated cyclization. This three-component reaction affords pharmaceutically valuable 4-aminoquinolines in a one-pot procedure from readily available starting materials. The reaction tolerates various substituents on the arene as well as the use of secondary and even primary isocyanides. Additionally, the wide tolerance for functionalized isocyanides allows for the one-pot synthesis of various substituted chloroquine analogues as well as other medicinally relevant products.

\section{INTRODUCTION}

4-Aminoquinolines (4-AQs) have been widely investigated for their pharmaceutical potential, in particular, their antimalarial properties. $^{1-5}$ Recently, B-ring-substituted (in particular, 7halo) 4-AQs were shown to display enhanced activities against highly resistant strains of Plasmodium falciparum. ${ }^{6}$ Consequently, this scaffold is still of high interest for the development of novel antimalarials. ${ }^{7}$ Additionally, chloroquine and amodiaquine (Figure 1) have recently been flagged as<smiles>[R1]Cc1cc([Al])nc2cc(N([R])[R])c([Y10])cc12</smiles><smiles>[R]Nc1cc(-c2ccc3ccccc3c2)nc2ccccc12</smiles>

Figure 1. Examples of medicinally relevant 4-aminoquinolines.

leads against Ebola and Marburg viruses. ${ }^{8,9}$ These known antimalarials also inhibit endosomal Toll-like receptors (TLRs). ${ }^{10}$ Recently, other applications of 4-AQs have also been reported, as diversely substituted 4-AQs have been identified as promising antifilarials, ${ }^{11}$ translocator protein biomarker ligands, ${ }^{12}$ tuberculosis ATP synthase inhibitors, ${ }^{13}$ and as nociceptin receptor antagonists. ${ }^{14,15}$ Additionally, 4-
AQs bearing 2-(hetero)aryl functionalities also show promise as non-nucleoside HIV-1 inhibitors. ${ }^{16}$ Not surprisingly, there is great interest to efficiently access the 4-AQ core, and over the past decades, several cascade reactions toward these medicinally valuable scaffolds have been developed (Scheme 1). ${ }^{17-22}$ For example, 4-AQs can be obtained from the reaction of ynamides and nitrilium species, resulting from either arylation of nitriles ${ }^{17}$ or dehydration of anilides. ${ }^{18}$ The $\mathrm{Cu}(\mathrm{I})$-catalyzed cyclization of imidoylacetylenes and sulfonyl azides affords 4AQs, as well. ${ }^{19}$ A carbonylative Sonogashira reaction of 2ethynylanilines with in situ amination at $\mathrm{C} 4$ also provides access to 4-AQs. ${ }^{21}$ Finally, we recently showed that palladiumcatalyzed oxidative isocyanide insertion of $N$-aryl imines by double $\mathrm{C}-\mathrm{H}$ activation also affords 4 -AQs. ${ }^{22}$ However, these methods rely on rather specialized starting materials ${ }^{17-20}$ and/ or afford the desired 4-AQs in disappointing yields. ${ }^{18,22}$ Most diversity-oriented routes toward 4-AQs rely on the amination of 4- haloquinolines, ${ }^{23,24}$ in turn derived from the corresponding 4-quinolones. Such multisubstituted quinolones (2) are generally difficult to synthesize by traditional condensation methods. $^{25-29}$ However, quinolones $\mathbf{2}$ are accessible via a carbonylative Sonogashira coupling (Scheme 2), using organic base to mediate the postcoupling cyclization (Scheme 2$).{ }^{30-32}$ To the best of our knowledge, only one example of isocyanide insertion in a Sonogashira coupling has been reported, where the product ynimines are hydrolyzed in situ to the corresponding ynones. $^{33,34}$ Based on this, we envisioned a direct, one-pot synthesis of 4-AQs 4 via an imidoylative Sonogashira coupling and subsequent cycloaromatization

Received: November 9, 2017

Published: December 20, 2017 
Scheme 1. Previous Routes to 4-Aminoquinolines

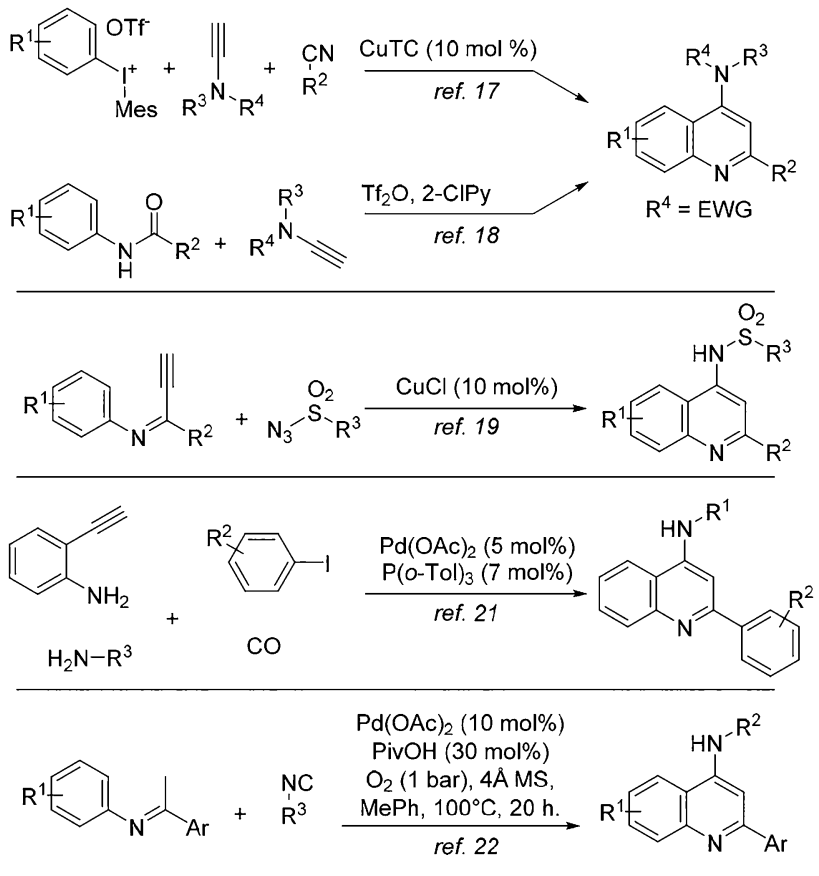

Scheme 2. Carbonylative and Imidoylative Sonogashira Coupling to 4-Aminoquinolines

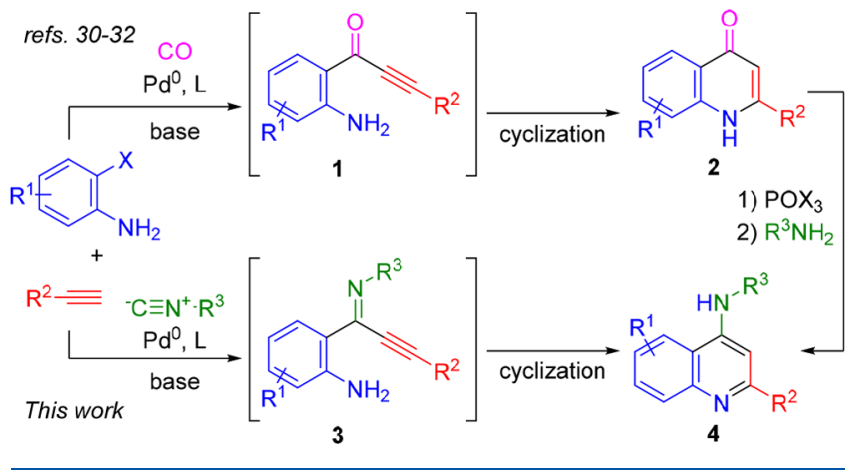

(Scheme 2). Such an approach prevents the need for toxic CO gas and avoids the two-step derivatization of quinolones 2 .

\section{RESULTS AND DISCUSSION}

Our initial studies showed that such an imidoylative Sonogashira approach using 5, 6a, and $7 \mathbf{a}$ afforded a range of products as a difficult to analyze mixture under typical conditions (Scheme 3). Most notably, with temperatures

Scheme 3. Initial Imidoylative Sonogashira Studies toward 4-AQs and Side Products<smiles>[X]c1ccccc1N</smiles>

$\mathrm{Pd}^{\mathrm{O}}$, Ligand, CuX, base

$6 \mathrm{a} \equiv \mathrm{Ph} \quad$ solvent, $16 \mathrm{~h}$

7a $-C \equiv N^{+}-t-B u$<smiles>CC(C)(C)N=C(C#Cc1ccccc1)c1ccccc1N</smiles><smiles>CC(C)(C)Nc1cc(-c2ccccc2)nc2ccccc12</smiles>

9<smiles>C(C#Cc1ccccc1)#Cc1ccccc1</smiles>

10<smiles>CC(C)(C)N=C1Nc2ccccc2/C1=N/C(C)(C)C</smiles>

under $50{ }^{\circ} \mathrm{C}$ or $\mathrm{CuBr}$ loadings approaching stoichiometric amounts, only the alkyne homocoupling product 10 was isolated. Additionally, SFC-MS analysis indicated that the use of $o$-iodoaniline promotes intramolecular Buchwald-Hartwigtype reactivity with double isocyanide insertion, resulting in the formation of isatin diimine 11 (Scheme 3). If $o$ bromoaniline is employed, formation of $\mathbf{1 1}$ is suppressed to trace amounts, which is known for the synthesis of these isatinimines. ${ }^{35}$ Surprisingly, these initial experiments revealed that the main product in all cases was the intermediate imidoylative Sonogashira product 8, with only trace amounts of the desired cyclization product 9. Direct formation of 4-AQs 9 from these previously underexplored ynimines 8 appears to be surprisingly difficult. Consequently, we focused our attention on the optimization of this cycloaromatization. Multiple cyclization conditions were investigated for the formation of aminoquinoline 9 from the proposed intermediate ynimine $\mathbf{8}$. Neither strong nor weak bases proved effective in facilitating this cyclization (Table 1, entries 1 and

\section{Table 1. Ynimine Cyclization Optimization}

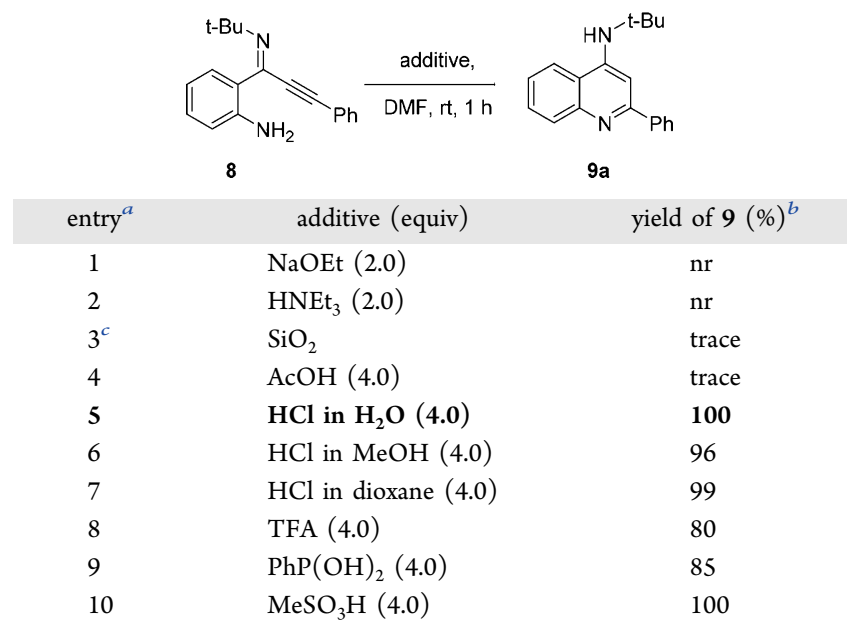

${ }^{a}$ Conditions: ynimine $8(0.55 \mathrm{mmol})$ in DMF ( $\left.1 \mathrm{~mL}\right)$, additive, $\mathrm{rt}, 1$ h. ${ }^{b}$ Yield determined by NMR using an internal standard $(1,3,5$ trimethoxybenzene). ${ }^{c}$ Using $0.150 \mathrm{~g}$ of $\mathrm{SiO}_{2} ; \mathrm{nr}=$ no reaction, $\mathrm{TFA}=$ trifluoroacetic acid.

2). Weak acids $\left(\mathrm{SiO}_{2}, \mathrm{AcOH}\right)$ were applied to activate the imine toward cyclization but only afforded cyclization product 9a in trace quantities (entries 3 and 4). However, stronger acids like TFA $\left(\mathrm{p} K_{\mathrm{a}}=0.23\right)$, phenylphosphinic acid $\left(\mathrm{p} K_{\mathrm{a}}=\right.$ $1.85)$, and methanesulfonic acid $\left(\mathrm{p} K_{\mathrm{a}}=-1.9\right)$ rapidly afforded 4-aminoquinoline 9a, even after short reaction times at ambient temperature (Table 1, entries 8-10). Interestingly, $\mathrm{HCl}$ also facilitated the cycloaromatization, even when employed as an aqueous solution. Curiously, acids with nonnucleophilic conjugate bases also afford aminoquinoline 9a in high to quantitative yield (Table 1 , entries 8 and 10), implying either an intermolecular Michael/Michael/retro-Michael mechanism ${ }^{30-32}$ or a direct intramolecular Michael reaction. Regardless, postcoupling addition of aqueous hydrochloric acid was selected as the optimal cyclization condition. While the addition of $1 \mathrm{M}$ aqueous $\mathrm{HCl}$ readily facilitated cycloaromatization of $\mathbf{8}$ to $9 \mathrm{a}$, we opted for slightly harsher conditions ( $2 \mathrm{M}$ aqueous $\mathrm{HCl}(4 \mathrm{~mL}), 15 \mathrm{~min}, \mathrm{rt}$ ) for the in situ cyclization following the imidoylative Sonogashira reaction to account for excess $\mathrm{Cs}_{2} \mathrm{CO}_{3}$ as well as any residual starting 
materials. With the optimized cyclization protocol in hand, we turned our attention to the optimization of the imidoylative Sonogashira coupling. Using standard conditions $(5 \mathrm{~mol} \%$ of $\mathrm{Pd}\left(\mathrm{PPh}_{3}\right)_{4}, 15 \mathrm{~mol} \%$ of $\mathrm{CuBr}, 2.0$ equiv of $\mathrm{Cs}_{2} \mathrm{CO}_{3}, \mathrm{DMSO}$, $90{ }^{\circ} \mathrm{C}, 16 \mathrm{~h}$ ), we obtained the desired 4-AQ 9a in $61 \%$ yield (Table 1 , entry 2 ). When the $\mathrm{Cu}(\mathrm{I}) \mathrm{Br}$ cocatalyst was omitted, only isatin diimine $\mathbf{1 1}$ was obtained (Table 2, entry 1).

Table 2. Optimization of the Imidoylative Sonogashira/ Cyclization Conditions $^{a}$

\begin{tabular}{|c|c|c|c|c|c|}
\hline \multirow{3}{*}{$\begin{array}{r}7 a \\
\text { entry }\end{array}$} & \multirow{2}{*}{$\begin{array}{l}{ }_{\mathrm{CNH}}^{\mathrm{Br}}-\mathrm{t}-\mathrm{Bu} \\
\equiv-\mathrm{Ph}\end{array}$} & \multicolumn{3}{|c|}{$\begin{array}{l}\text { 1) } \mathrm{Pd}(\mathrm{OAC})_{2}(5 \mathrm{~mol} \%) \text {, ligand } \\
\mathrm{CuBr}(15 \mathrm{~mol} \%), \mathrm{Cs}_{2} \mathrm{CO}_{3}(2.0 \mathrm{eq}) \text {, } \\
\text { solvent, } 90^{\circ} \mathrm{C}, 16 \mathrm{~h}\end{array}$} & \multirow{2}{*}{$\mathrm{N}_{\mathrm{Ph}}$} \\
\hline & & 2) $2 \mathrm{I}$ & tt, $15 \mathrm{~min}$ & & \\
\hline & & ource & ligand & solvent & 9a $(\%)^{b}$ \\
\hline $1^{c}$ & & $\left.\mathrm{Ph}_{3}\right)_{4}$ & none & DMSO & $\mathrm{nr}$ \\
\hline 2 & & $\left.\mathrm{Ph}_{3}\right)_{4}$ & none & DMSO & 61 \\
\hline 3 & & $\left.\mathrm{Ph}_{3}\right)_{4}$ & none & toluene & 27 \\
\hline 4 & & $\left.\mathrm{Ph}_{3}\right)_{4}$ & none & dioxane & 13 \\
\hline 5 & & $\left.\mathrm{Ph}_{3}\right)_{4}$ & none & DMF & 70 \\
\hline 6 & $\mathrm{Pd}$ & & none & DMF & $\mathrm{nr}$ \\
\hline 7 & & $\mathrm{Ac})_{2}$ & $\mathrm{PPh}_{3}$ & DMF & 82 \\
\hline 8 & & Ac) $)_{2}$ & $\mathrm{Bu}_{3} \mathrm{P}$ & DMF & $\mathrm{nr}$ \\
\hline 9 & & $\mathrm{Ac})_{2}$ & DPEPhos & DMF & 82 \\
\hline 10 & & AAc) $)_{2}$ & XantPhos & DMF & 91 \\
\hline $11^{d}$ & & Ac) $)_{2}$ & XantPhos & DMF & $\mathrm{nr}$ \\
\hline $12^{e}$ & & Ac) $)_{2}$ & XantPhos & DMF & 56 \\
\hline $13^{f}$ & & Ac) ${ }_{2}$ & XantPhos & DMF & $\mathrm{nr}$ \\
\hline $14^{g}$ & & Ac) ${ }_{2}$ & XantPhos & DMF & 31 \\
\hline 15 & & Ac) $)_{2}$ & none & DMF & $\mathrm{nr}$ \\
\hline 16 & nor & & XantPhos & DMF & $\mathrm{nr}$ \\
\hline
\end{tabular}

${ }^{a}$ Reaction conditions: 2-bromoaniline (12a, $0.5 \mathrm{mmol}, 1$ equiv), phenylacetylene (6a, $1 \mathrm{mmol}, 2$ equiv), tert-butyl isocyanide ( $7 \mathrm{a}$, $0.625 \mathrm{mmol}, 1.25$ equiv), catalyst ( $5 \mathrm{~mol} \%)$, ligand (monodentate: 15 mol \%, bidentate: $10 \mathrm{~mol} \%), \mathrm{CuBr}(15 \mathrm{~mol} \%)$, and $\mathrm{Cs}_{2} \mathrm{CO}_{3}(1$ mmol, 2 equiv) in solvent $(3.0 \mathrm{~mL})$ were stirred at $100{ }^{\circ} \mathrm{C}$ for $16-20$ $\mathrm{h}$ under $\mathrm{N}_{2}$ atmosphere. ${ }^{b}$ Yields determined by ${ }^{1} \mathrm{H}$ NMR analysis using 2,5-dimethylfuran as internal standard. ${ }^{c}$ Reaction performed in the absence of $\mathrm{CuBr} .{ }^{d} \mathrm{KO} t \mathrm{Bu}$ (2.0 equiv) employed as base. ${ }^{e} \mathrm{~K}_{2} \mathrm{CO}_{3}$ (2.0 equiv) employed as base. ${ }^{f} \mathrm{Et}_{3} \mathrm{~N}$ (2.0 equiv) employed as base. ${ }^{g} \mathrm{DBU}$ (2.0 equiv) employed as base.

Subsequent solvent screening indicated that the transformation proceeds most efficiently in polar aprotic solvents, with DMF giving superior results (entries 2-5). As expected, a heterogeneous palladium catalyst $(\mathrm{Pd} / \mathrm{C})$ did not catalyze the reaction (entry 6). Switching from $\mathrm{Pd}\left(\mathrm{PPh}_{3}\right)_{4}$ to an in situ formed $\mathrm{Pd}^{0}$ complex allowed for a ligand screening (entries 710). Interestingly, in addition to leading to the highest 4-AQ yield, the use of Xantphos as a ligand completely inhibited the formation of isatin diimine 11 (Table 1, entry 10). Changing the base from $\mathrm{Cs}_{2} \mathrm{CO}_{3}$ to other inorganic or organic bases $\left(\mathrm{KO} t \mathrm{Bu}, \mathrm{K}_{2} \mathrm{CO}_{3}, \mathrm{Et}_{3} \mathrm{~N}, \mathrm{DBU}\right)$ did not lead to an increase in yield (entries 11-14). Finally, as anticipated, 9a was not formed when either the palladium source or the ligand was omitted (entries 15 and 16).

With the optimized reaction conditions in hand, we set out to explore the compatibility of diversely substituted $o$ bromoanilines and terminal alkynes with our three-component reaction (Scheme 4). The reaction proved compatible with neutral and moderately electron-donating substituents $(\mathbf{9 a}, \mathbf{b})$. The use of halo-substituted $o$-bromoanilines did not lead to
Scheme 4. Alkyne and o-Bromoaniline Variation ${ }^{a}$

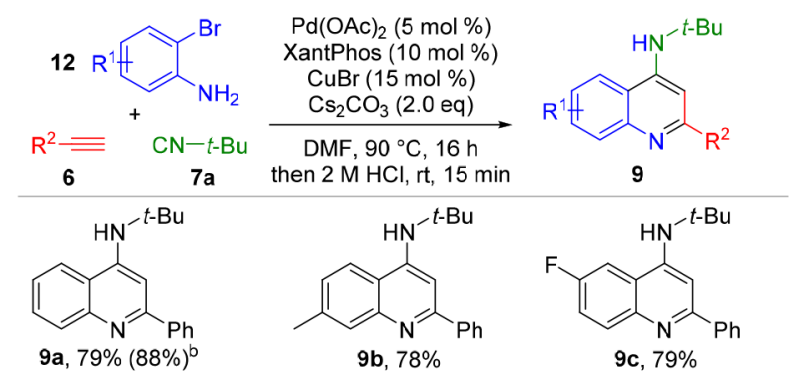<smiles>Cc1ccc(-c2cc(NC(C)(C)C)c3cc(F)ccc3n2)cc1</smiles><smiles>COc1ccc(-c2cc(NC(C)(C)C)c3ccc(F)cc3n2)cc1</smiles><smiles>COc1cc(-c2cc(NCC(C)(C)C)c3ccc(Cl)cc3n2)cc(-c2cc(NC(C)(C)C)c3ccccc3n2)c1</smiles><smiles>CCCCc1cc(NC(C)(C)C)c2ccccc2n1</smiles>

${ }^{a}$ Conditions: $o$-bromoaniline $(12,1.0 \mathrm{mmol})$, tert-butyl isocyanide (7a, $1.25 \mathrm{mmol})$, alkyne $(6,2.0 \mathrm{mmol}), \mathrm{Pd}(\mathrm{OAc})_{2}(0.05 \mathrm{mmol})$, Xantphos (0.1 mmol), $\mathrm{CuBr}(0.15 \mathrm{mmol}), \mathrm{Cs}_{2} \mathrm{CO}_{3}(2.0 \mathrm{mmol})$ in DMF $(8.0 \mathrm{~mL}) .{ }^{b}$ Reaction performed on $10.0 \mathrm{mmol}$ scale. ${ }^{c}$ Closed vial, $5.0 \mathrm{mmol}$ of 1-hexyne.

lower yields $(9 \mathrm{c}-\mathrm{g})$. Decoration of the $o$-bromoaniline or the arylalkyne with electron-donating functionalities afforded the substituted 4-AQs in high yields $(9 \mathbf{h}-\mathbf{k})$. However, obromoanilines bearing electron-withdrawing substituents displayed lower compatibility with the three-component cascade protocol. When 3-bromo-4-aminobenzoate was used, the corresponding aminoquinoline 91 was isolated in lower yield. Similar results were observed for $\mathbf{9 m}$, where the introduction of an additional distal electron acceptor on the opposite side of the ynimine renders the alkyne susceptible to 5-exo-dig cyclization. This cyclization to an aza-aurone side product most likely accounts for the diminished yields, as it does not require external acid activation and can therefore take place during the reaction. Additionally, it is possible that withdrawing electron density through substituent effects renders ynimines $\mathbf{8}$ more susceptible to hydrolysis during the cyclization stage. Gratifyingly, the developed conditions proved to tolerate aliphatic alkynes, as well, as demonstrated by the isolation of $\mathbf{9 n}$ and $\mathbf{9 0}$, with somewhat lower yield for $\mathbf{9 n}$ as a result of the volatility of 1-hexyne.

Interestingly, this palladium-catalyzed imidoylative Sonogashira coupling displayed high tolerance with regard to the isocyanide input (Scheme 5). Secondary isocyanides, which are 


\section{Scheme 5. Isocyanide Variation ${ }^{a}$}

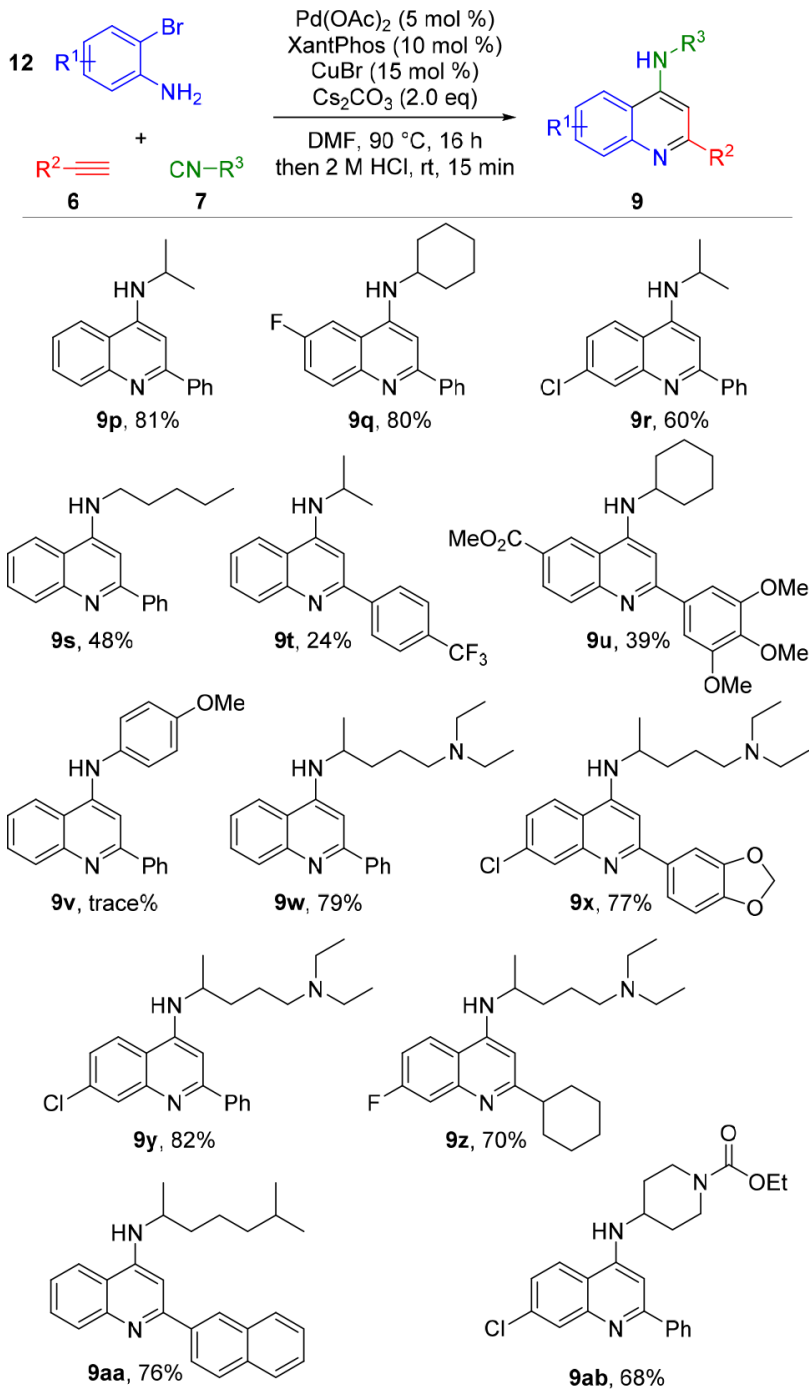

${ }^{a}$ Conditions: $o$-bromoaniline $(1.0 \mathrm{mmol})$, isocyanide $(1.75 \mathrm{mmol})$, alkyne (2.0 mmol), $\mathrm{Pd}(\mathrm{OAc})_{2}(0.05 \mathrm{mmol})$, Xantphos $(0.1 \mathrm{mmol})$, $\mathrm{CuBr}(0.15 \mathrm{mmol}), \mathrm{Cs}_{2} \mathrm{CO}_{3}(2.0 \mathrm{mmol})$ in $\mathrm{DMF}(8.0 \mathrm{~mL})$.

known to undergo double insertion more readily under palladium catalysis, ${ }^{36,37}$ proved fully compatible with this protocol $(9 \mathbf{p}-\mathbf{r})$. Satisfyingly, even primary isocyanide insertion afforded aminoquinoline $9 \mathrm{~s}$ in acceptable yield. The use of bromoanilines and arylalkynes bearing electron-withdrawing substituents afforded the corresponding products in lower yields, in accordance with earlier results $(9 t, \mathbf{u})$. Unfortunately, the use of 4-methoxyphenyl isocyanide did not afford isolable quantities of the corresponding 4arylaminoquinoline $9 \mathbf{v}$. We then investigated the utility of our method in the synthesis of pharmaceutically relevant 4AQs. The use of readily accessible N,N-diethyl-4-isocyanopentan-1-amine provided access to a variety of chloroquine analogues $(\mathbf{9 w}-\mathbf{9 y})$ in high yields. Similarly, the use of the isocyanide derived from the known vasoconstrictor octodrine ${ }^{38}$ afforded 9aa in good yield. Analogues of this highly fluorescent 2-naphthyl-4-AQ have been identified as potent immunostimulatory CpG-oligonucleotide antagonists. ${ }^{39,40}$ Furthermore, the use of 2-bromo-5-fluoroaniline, combined with $N, N$ diethyl-4-isocyanopentan-1-amine and cyclohexylacetylene, furnishes 4-amino-2-cyclohexyl-7-fluoroquinoline (9z). Struc- tures of this type have been described as promising antileishmanials. $^{41}$ Finally, ethyl 4-isocyanopiperidine-1-carboxylate can be used to construct $9 \mathrm{ab}$ in $68 \%$ yield, providing a handle for further functionalization of these medicinally valuable scaffolds.

In conclusion, we have developed a novel one-pot imidoylative Sonogashira cross-coupling/acid-mediated cyclization toward 4-aminoquinolines. The devised methodology is highly compatible with electronically diverse $o$-bromoanilines, arylalkynes, and aliphatic alkynes, although electron-withdrawing substituents on either arene lead to lower yields. The transformation is compatible with tertiary, secondary, and even primary aliphatic isocyanides. Additionally, using functionalized alkyl isocyanides, this method can be used to directly synthesize 2-substituted 4-aminoquinolines of high medicinal relevance.

\section{EXPERIMENTAL SECTION}

General Information. Chemicals were purchased from SigmaAldrich or Fluorochem and were used without purification. Solvents were purchased from VWR Chemicals $\left(\mathrm{CH}_{2} \mathrm{Cl}_{2}\right)$ or Sigma-Aldrich (toluene, dioxane, DMSO) and used without purification, unless stated otherwise. Dry solvents were dried over an inert PS-MD-5 solvent purification system, equipped with an activated alumina/ copper wire column. ${ }^{1} \mathrm{H}$ NMR measurements were acquired on a Bruker Avance 300 (300.13 MHz) or Bruker Avance 500 (500.23 $\mathrm{MHz})$ spectrometer. ${ }^{13} \mathrm{C}$ NMR measurements were acquired on a Bruker Avance $500(125.78 \mathrm{MHz})$ spectrometer. Chemical shifts are reported in parts per million downfield of tetramethylsilane and are corrected according to solvent. Mass analysis was performed using a Bruker MicrOTOF- $Q$ instrument on a positive ion polarity mode for ESI (electrospray ionization). Capillary charge: 4000 V. Melting points were measured using a Büchi M-565 melting point apparatus. $\mathrm{SiO}_{2}$ column chromatography was performed using Merck silica gel C60 (particle size 40-60 $\mu \mathrm{m}$ ). TLC chromatography was performed on Merck silica gel C60 F254 plates (silica coat on aluminum support). All isolated yields are corrected for present impurities (if present).

General Procedure 1 for the Synthesis of 4-tert-Butylaminoquinolines from Bromoanilines, Alkynes, and tert-Butyl Isocyanide. A solution of $\mathrm{Pd}(\mathrm{OAc})_{2}(0.011 \mathrm{~g}, 0.05 \mathrm{mmol})$ and Xantphos $(0.058 \mathrm{~g}, 0.10 \mathrm{mmol})$ in dry DMF $(8 \mathrm{~mL})$ was stirred for 20 min at $\mathrm{rt}$ under $\mathrm{N}_{2}$ atmosphere, forming a yellow suspension. To this mixture were added consecutively $\mathrm{Cs}_{2} \mathrm{CO}_{3}(0.651 \mathrm{~g}, 2.0 \mathrm{mmol})$, obromoaniline $(1.0 \mathrm{mmol})$, tert-butyl isocyanide $(0.141 \mathrm{~mL}, 1.25$ $\mathrm{mmol}), \mathrm{CuBr}(0.021 \mathrm{~g}, 0.15 \mathrm{mmol})$, and alkyne $(2.0 \mathrm{mmol})$. The resulting mixture was stirred at $90{ }^{\circ} \mathrm{C}$ for $16 \mathrm{~h}$. Hereafter, the reaction was allowed to cool to rt, after which $4 \mathrm{~mL}$ of $2 \mathrm{M} \mathrm{HCl}$ was added and stirred for $15 \mathrm{~min}$. The mixture was diluted with $\mathrm{CH}_{2} \mathrm{Cl}_{2}$, filtered over a pad of diatomaceous earth, and washed with $1 \mathrm{M} \mathrm{HCl}$ and saturated $\mathrm{NaHCO}_{3}$, before being dried over anhydrous $\mathrm{Na}_{2} \mathrm{SO}_{4}$, filtered, and concentrated in vacuo. Purification was performed by column chromatography (gradient, $\mathrm{cHex} / \mathrm{EtOAc} / \mathrm{Et}_{3} \mathrm{~N}$ 19:1:0.01-1:1:0.01, unless stated otherwise) to afford the pure 4-aminoquinoline derivative.

General Procedure 2 for the Synthesis of 4-Aminoquinolines from Bromoanilines, Alkynes, and Isocyanides. A solution of $\mathrm{Pd}(\mathrm{OAc})_{2}(0.011 \mathrm{~g}, 0.05 \mathrm{mmol})$ and Xantphos $(0.058 \mathrm{~g}, 0.10$ $\mathrm{mmol})$ in dry DMF $(8 \mathrm{~mL})$ was stirred for 20 min at rt under $\mathrm{N}_{2}$ atmosphere, forming a yellow suspension. To this mixture were added consecutively $\mathrm{Cs}_{2} \mathrm{CO}_{3}(0.651 \mathrm{~g}, 2.0 \mathrm{mmol})$, o-bromoaniline (1.0 $\mathrm{mmol})$, isocyanide $(1.75 \mathrm{mmol}), \mathrm{CuBr}(0.021 \mathrm{~g}, 0.15 \mathrm{mmol})$, and alkyne $(2.0 \mathrm{mmol})$. The resulting mixture was stirred at $90{ }^{\circ} \mathrm{C}$ for 16 h. Hereafter, the reaction was allowed to cool to rt, after which $4 \mathrm{~mL}$ of $2 \mathrm{M} \mathrm{HCl}$ was added and stirred for $15 \mathrm{~min}$. The mixture was diluted with $\mathrm{CH}_{2} \mathrm{Cl}_{2}$, filtered over a pad of diatomaceous earth, and washed with $1 \mathrm{M} \mathrm{HCl}$ and saturated $\mathrm{NaHCO}_{3}$, before being dried over anhydrous $\mathrm{Na}_{2} \mathrm{SO}_{4}$, filtered, and concentrated in vacuo. 
Purification was performed by column chromatography (gradient, $\mathrm{cHex} / \mathrm{EtOAc} / \mathrm{Et}_{3} \mathrm{~N}$ 19:1:0.01-1:1:0.01, unless stated otherwise) to afford the pure 4-aminoquinoline derivative.

$\mathrm{N}$-(tert-Butyl)-2-phenylquinolin-4-amine (9a). Synthesized in accordance with general procedure 1 . Isolated as a yellow solid (0.234 g, 79\%): $R_{f}=0.19$ (cHex/EtOAc/Et ${ }_{3} \mathrm{~N} \mathrm{4:1:0.05);} \mathrm{mp} 139^{\circ} \mathrm{C}$; ${ }^{1} \mathrm{H}$ NMR $\left(500.23 \mathrm{MHz}, \mathrm{CDCl}_{3}\right) \delta 8.10-8.03(\mathrm{~m}, 3 \mathrm{H}), 7.68(\mathrm{dd}, J=$ $1.5,8.5 \mathrm{~Hz}, 1 \mathrm{H}), 7.63(\mathrm{ddd}, J=1.5,7.0,8.5 \mathrm{~Hz}, 1 \mathrm{H}), 7.51(\mathrm{t}, J=7.5$ $\mathrm{Hz}, 2 \mathrm{H}), 7.47-7.38(\mathrm{~m}, 2 \mathrm{H}), 7.12(\mathrm{~s}, 1 \mathrm{H}), 5.03(\mathrm{bs}, 1 \mathrm{H}), 1.60(\mathrm{~s}$, $9 \mathrm{H}) ;{ }^{13} \mathrm{C}$ NMR $\left(125.97 \mathrm{MHz}, \mathrm{CDCl}_{3}\right) \delta 157.9,148.8,148.3,141.2$, $130.5,129.0,128.8,128.6,127.5,124.3,118.9,118.5,99.1,51.5,29.4$; HRMS (ESI) $m / z$ calcd for $\mathrm{C}_{19} \mathrm{H}_{21} \mathrm{~N}_{2}{ }^{+}[\mathrm{M}+\mathrm{H}]^{+} 277.1695$, found 277.1707.

$\mathrm{N}$-(tert-Butyl)-7-methyl-2-phenylquinolin-4-amine (9b). Synthesized in accordance with general procedure 1. Isolated as a yellow solid $(0.237 \mathrm{~g}, 78 \%): R_{f}=0.33\left(\mathrm{cHex} / \mathrm{EtOAc} / \mathrm{Et}_{3} \mathrm{~N} 4: 1: 0.05\right) ; \mathrm{mp}$ $134{ }^{\circ} \mathrm{C}$; ${ }^{1} \mathrm{H}$ NMR $\left(500.23 \mathrm{MHz}, \mathrm{CDCl}_{3}\right) \delta 8.05$ (dd, $J=1.5,7.5 \mathrm{~Hz}$, $2 \mathrm{H}), 7.85(\mathrm{~s}, 1 \mathrm{H}), 7.57(\mathrm{~d}, J=8.5 \mathrm{~Hz}, 1 \mathrm{H}), 7.50(\mathrm{t}, J=7.5 \mathrm{~Hz}, 2 \mathrm{H})$, $7.47-7.40(\mathrm{~m}, 1 \mathrm{H}), 7.23(\mathrm{dd}, J=7.5,8.5 \mathrm{~Hz}, 1 \mathrm{H}), 7.07(\mathrm{~d}, J=1.5$ $\mathrm{Hz}, 1 \mathrm{H}), 4.99(\mathrm{bs}, 1 \mathrm{H}), 2.52(\mathrm{~s}, 3 \mathrm{H}), 1.59(\mathrm{~s}, 9 \mathrm{H}) ;{ }^{13} \mathrm{C}$ NMR $(125.97$ $\left.\mathrm{MHz}_{\mathrm{CDCl}}\right) \delta 157.9,149.0,148.3,141.3,139.0,129.7,128.7,128.6$, 127.5, 126.4, 118.6, 116.4, 98.7, 51.4, 29.4, 21.5; HRMS (ESI) $\mathrm{m} / z$ calcd for $\mathrm{C}_{20} \mathrm{H}_{23} \mathrm{~N}_{2}^{+}[\mathrm{M}+\mathrm{H}]^{+}$291.1851, found 291.1854.

$\mathrm{N}$-(tert-Butyl)-6-fluoro-2-phenylquinolin-4-amine (9c). Synthesized in accordance with general procedure 1 . Isolated as a light brown solid (0.232 g, 79\%): $R_{f}=0.44\left(\mathrm{cHex} / \mathrm{EtOAc} / \mathrm{Et}_{3} \mathrm{~N}\right.$ 4:1:0.05); $\mathrm{mp} 163{ }^{\circ} \mathrm{C}$; ${ }^{1} \mathrm{H} \mathrm{NMR}\left(500.23 \mathrm{MHz}, \mathrm{CDCl}_{3}\right) \delta 8.14-8.06(\mathrm{~m}, 3 \mathrm{H})$, $7.54(\mathrm{t}, J=7.5 \mathrm{~Hz}, 2 \mathrm{H}), 7.50-7.44(\mathrm{~m}, 1 \mathrm{H}), 7.48-7.37(\mathrm{~m}, 1 \mathrm{H})$, 7.36 (dd, $J=2.5,10.0 \mathrm{~Hz}, 1 \mathrm{H}), 7.17(\mathrm{~s}, 1 \mathrm{H}), 4.79$ (bs, $1 \mathrm{H}), 1.57$ (s, $9 \mathrm{H}) ;{ }^{13} \mathrm{C}$ NMR $\left(125.97 \mathrm{MHz}, \mathrm{CDCl}_{3}\right) \delta 159.5(\mathrm{~d}, J=245.0 \mathrm{~Hz})$, $157.3(\mathrm{~d}, J=2.5 \mathrm{~Hz}), 147.9$ (d, $J=4.5 \mathrm{~Hz}), 145.8,140.9,132.8(\mathrm{~d}, J=$ $8.5 \mathrm{~Hz}), 128.8,128.6,127.4,118.9(\mathrm{~d}, J=8.0 \mathrm{~Hz}), 118.6(\mathrm{~d}, J=24.5$ $\mathrm{Hz}), 103.3(\mathrm{~d}, J=23.0 \mathrm{~Hz}), 99.4,51.6,29.3$; HRMS (ESI) $\mathrm{m} / z$ calcd for $\mathrm{C}_{19} \mathrm{H}_{20} \mathrm{~N}_{2} \mathrm{~F}^{+}[\mathrm{M}+\mathrm{H}]^{+}$295.1601, found 295.1604.

$\mathrm{N}$-(tert-Butyl)-6-fluoro-2-(p-tolyl)quinolin-4-amine (9d). Synthesized in accordance with general procedure 1 . Isolated as a light brown solid $(0.241 \mathrm{~g}, 78 \%): R_{f}=0.43\left(\mathrm{cHex} / \mathrm{EtOAc} / \mathrm{Et}_{3} \mathrm{~N}\right.$ 4:1:0.05); $\mathrm{mp} 155{ }^{\circ} \mathrm{C}$; ${ }^{1} \mathrm{H}$ NMR $\left(500.23 \mathrm{MHz}, \mathrm{CDCl}_{3}\right) \delta 8.04$ (dd, $J=5.5,9.0$ $\mathrm{Hz}, 1 \mathrm{H}), 7.95(\mathrm{~d}, J=8.0 \mathrm{~Hz}, 2 \mathrm{H}), 7.42-7.36(\mathrm{~m}, 1 \mathrm{H}), 7.34-7.27(\mathrm{~m}$, $3 \mathrm{H}), 7.12$ (s, $1 \mathrm{H}), 4.74$ (bs, $1 \mathrm{H}), 2.43(\mathrm{~s}, 3 \mathrm{H}), 1.59(\mathrm{~s}, 9 \mathrm{H}) ;{ }^{13} \mathrm{C}$ NMR $\left(125.97 \mathrm{MHz}, \mathrm{CDCl}_{3}\right) \delta 159.5(\mathrm{~d}, J=245.0 \mathrm{~Hz}), 157.3(\mathrm{~d}, J=$ $2.5 \mathrm{~Hz}), 147.8$ (d, $J=5.0 \mathrm{~Hz}), 145.8,138.8,138.0,132.7(\mathrm{~d}, J=8.5$ $\mathrm{Hz}), 129.4,127.3,118.9$ (d, $J=8.0 \mathrm{~Hz}), 118.5(\mathrm{~d}, J=25.0 \mathrm{~Hz}), 103.3$ (d, $J=23.0 \mathrm{~Hz}$ ), 99.3, 51.5, 29.3, 21.3; HRMS (ESI) $\mathrm{m} / z$ calcd for $\mathrm{C}_{20} \mathrm{H}_{22} \mathrm{~N}_{2} \mathrm{~F}^{+}[\mathrm{M}+\mathrm{H}]^{+}$309.1757, found 309.1769.

$\mathrm{N}$-(tert-Butyl)-6-chloro-8-fluoro-2-(p-tolyl)quinolin-4-amine (9e). Synthesized in accordance with general procedure 1 . Isolated as a yellow solid (0.253 g, 78\%): $R_{f}=0.69\left(\mathrm{cHex} / \mathrm{EtOAc} / \mathrm{Et}_{3} \mathrm{~N}\right.$ 4:1:0.01); mp 166-167 ${ }^{\circ} \mathrm{C} ;{ }^{1} \mathrm{H}$ NMR $\left(500.23 \mathrm{MHz}, \mathrm{CDCl}_{3}\right) \delta 7.98$ (dd, $J=8.0$, $2.0 \mathrm{~Hz}, 1 \mathrm{H}), 7.42(\mathrm{~s}, 1 \mathrm{H}), 7.34-7.28(\mathrm{~m}, 3 \mathrm{H}), 7.17(\mathrm{~s}, 1 \mathrm{H}), 4.86(\mathrm{~s}$, 0H), $2.42(\mathrm{~s}, 3 \mathrm{H}), 1.59$ (s, 9H); ${ }^{13} \mathrm{C}$ NMR $\left(125.97 \mathrm{MHz}, \mathrm{CDCl}_{3}\right) \delta$ $159.8,157.8(\mathrm{~d}, J=18.5 \mathrm{~Hz}), 147.3(\mathrm{~d}, J=3.5 \mathrm{~Hz}), 138.4(\mathrm{~d}, J=$ $235.5 \mathrm{~Hz}), 138.2(\mathrm{~d}, J=11.0 \mathrm{~Hz}), 129.4,128.5(\mathrm{~d}, J=11.0 \mathrm{~Hz})$, 127.37, $120.6(\mathrm{~d}, J=3.5 \mathrm{~Hz}), 114.7(\mathrm{~d}, J=23.0 \mathrm{~Hz}), 114.4(\mathrm{~d}, J=4.5$ $\mathrm{Hz}$ ), 100.1, 51.8, 29.3, 21.4; HRMS (ESI) $\mathrm{m} / \mathrm{z}$ calcd for $\mathrm{C}_{20} \mathrm{H}_{21} \mathrm{~N}_{2} \mathrm{ClF}^{+}[\mathrm{M}+\mathrm{H}]^{+}$343.1368, found 343.1376.

$\mathrm{N}$-(tert-Butyl)-7-chloro-2-(3-chlorophenyl)quinolin-4-amine (9f). Synthesized in accordance with general procedure 1 . Isolated as a yellow solid $(0.231 \mathrm{~g}, 67 \%): R_{f}=0.58\left(\mathrm{cHex} / \mathrm{EtOAc} / \mathrm{Et}_{3} \mathrm{~N}\right.$ 4:1:0.01); mp 51-54 ${ }^{\circ} \mathrm{C}$; ${ }^{1} \mathrm{H}$ NMR $\left(500.23 \mathrm{MHz}, \mathrm{CDCl}_{3}\right) \delta 8.08-7.98(\mathrm{~m}$, $2 \mathrm{H}), 7.89(\mathrm{~d}, J=6.5 \mathrm{~Hz}, 1 \mathrm{H}), 7.59(\mathrm{~d}, J=9.0 \mathrm{~Hz}, 1 \mathrm{H}), 7.47-7.39$ $(\mathrm{m}, 2 \mathrm{H}), 7.34(\mathrm{~d}, J=9.0 \mathrm{~Hz}, 1 \mathrm{H}), 7.04(\mathrm{~s}, 1 \mathrm{H}), 5.03(\mathrm{bs}, 1 \mathrm{H}), 1.59$ $(\mathrm{s}, 9 \mathrm{H}) ;{ }^{13} \mathrm{C}$ NMR $\left(125.97 \mathrm{MHz}, \mathrm{CDCl}_{3}\right) \delta 157.3,149.5,148.6$, $142.5,135.0,134.7,129.9,129.3,129.0,127.6,125.5,125.2,120.5$, 117.0, 98.9, 51.7, 29.3; HRMS (ESI) $m / z$ calcd for $\mathrm{C}_{19} \mathrm{H}_{19} \mathrm{~N}_{2} \mathrm{Cl}_{2}^{+}[\mathrm{M}$ $+\mathrm{H}]^{+}$345.0917, found 345.0925.

$\mathrm{N}$-(tert-Butyl)-2-(4-fluorophenyl)quinolin-4-amine (9g). Synthesized in accordance with general procedure 1. Isolated as a yellow solid $(0.252 \mathrm{~g}, 77 \%): R_{f}=0.33\left(\mathrm{cHex} / \mathrm{EtOAc} / \mathrm{Et}_{3} \mathrm{~N} \mathrm{4:1:0.05); \textrm {mp }}\right.$ $138{ }^{\circ} \mathrm{C} ;{ }^{1} \mathrm{H}$ NMR $\left(500.23 \mathrm{MHz}, \mathrm{CDCl}_{3}\right) \delta 8.09-8.01(\mathrm{~m}, 3 \mathrm{H}), 7.68$ $(\mathrm{d}, J=8.5 \mathrm{~Hz}, 1 \mathrm{H}), 7.67-7.60(\mathrm{~m}, 1 \mathrm{H}), 7.44-7.37(\mathrm{~m}, 1 \mathrm{H}), 7.19(\mathrm{t}$, $J=8.5 \mathrm{~Hz}, 2 \mathrm{H}), 7.06(\mathrm{~s}, 1 \mathrm{H}), 5.05(\mathrm{bs}, 1 \mathrm{H}), 1.60(\mathrm{~s}, 9 \mathrm{H}) ;{ }^{13} \mathrm{C} \mathrm{NMR}$ $\left(125.97 \mathrm{MHz}, \mathrm{CDCl}_{3}\right) \delta 163.4(\mathrm{~d}, J=248.0 \mathrm{~Hz}), 156.8,148.8,148.4$, $137.3(\mathrm{~d}, J=3.0 \mathrm{~Hz}), 130.4,129.2(\mathrm{~d}, J=8.5 \mathrm{~Hz}), 129.1,124.4$, $118.9,118.4,115.5(\mathrm{~d}, J=21.5 \mathrm{~Hz}), 98.7$; HRMS (ESI) $\mathrm{m} / z$ calcd for $\mathrm{C}_{19} \mathrm{H}_{20} \mathrm{~N}_{2} \mathrm{~F}^{+}[\mathrm{M}+\mathrm{H}]^{+}$295.1601, found 295.1601.

$\mathrm{N}$-(tert-Butyl)-7-fluoro-2-(4-methoxyphenyl)quinolin-4-amine (9h). Synthesized in accordance with general procedure 1 . Isolated as a yellow solid $(0.257 \mathrm{~g}, 79 \%): R_{f}=0.38\left(\mathrm{cHex} / \mathrm{EtOAc} / \mathrm{Et}_{3} \mathrm{~N}\right.$ 4:1:0.01); mp 47-49 ${ }^{\circ} \mathrm{C} ;{ }^{1} \mathrm{H}$ NMR (500.23 MHz, $\left.\mathrm{CDCl}_{3}\right) \delta 8.11-$ $7.99(\mathrm{~m}, 2 \mathrm{H}), 7.78-7.57(\mathrm{~m}, 2 \mathrm{H}), 7.13(\mathrm{t}, J=8.5 \mathrm{~Hz}, 1 \mathrm{H}), 7.08-$ $6.93(\mathrm{~m}, 3 \mathrm{H}), 4.97(\mathrm{bs}, 1 \mathrm{H}), 3.88(\mathrm{~s}, 3 \mathrm{H}), 1.59(\mathrm{~s}, 9 \mathrm{H}) ;{ }^{13} \mathrm{C} \mathrm{NMR}$ $\left(125.97 \mathrm{MHz}, \mathrm{CDCl}_{3}\right) \delta 163.0(\mathrm{~d}, J=248.0 \mathrm{~Hz}), 160.5,158.5,150.3$ $(\mathrm{d}, J=12 \mathrm{~Hz}), 148.4,133.3,128.8,121.1(\mathrm{~d}, J=10.0 \mathrm{~Hz}), 114.0$, 113.7 (d, $J=19.5 \mathrm{~Hz}$ ), 113.6 (d, $J=25.0 \mathrm{~Hz}$ ), 98.3, 55.4, 51.6, 29.4); HRMS (ESI) $m / z$ calcd for $\mathrm{C}_{20} \mathrm{H}_{22} \mathrm{~N}_{2} \mathrm{FO}^{+}[\mathrm{M}+\mathrm{H}]^{+} 325.1706$, found 325.1723.

$\mathrm{N}$-(tert-Butyl)-7-methoxy-2-phenylquinolin-4-amine (9i). Synthesized in accordance with general procedure 1 . Isolated as a bright yellow solid $(0.236 \mathrm{~g}, 77 \%): R_{f}=0.29\left(\mathrm{cHex} / \mathrm{EtOAc} / \mathrm{Et}_{3} \mathrm{~N} 4: 1: 0.01\right)$; mp 56-61 ${ }^{\circ} \mathrm{C} ;{ }^{1} \mathrm{H}$ NMR $\left(500.23 \mathrm{MHz}, \mathrm{CDCl}_{3}\right) \delta 8.06(\mathrm{~m}, 2 \mathrm{H}), 7.57$ $(\mathrm{d}, J=9.0 \mathrm{~Hz}, 1 \mathrm{H}), 7.54-7.47(\mathrm{~m}, 2 \mathrm{H}), 7.46-7.41(\mathrm{~m}, 2 \mathrm{H}), 7.04$ (dd, $J=2.5,9.0 \mathrm{~Hz}, 1 \mathrm{H}), 7.02 \mathrm{~s}, 1 \mathrm{H}), 4.96(\mathrm{bs}, 1 \mathrm{H}), 3.94(\mathrm{~s}, 3 \mathrm{H})$, $1.58(\mathrm{~s}, 9 \mathrm{H}) ;{ }^{13} \mathrm{C}$ NMR $\left(125.97 \mathrm{MHz}, \mathrm{CDCl}_{3}\right) \delta 160.2,158.4,150.7$, $148.5,141.3,128.7,128.6,127.4,120.2,116.7,112.9,108.6,98.2$, 55.4, 51.5, 29.4; HRMS (ESI) $m / z$ calcd for $\mathrm{C}_{20} \mathrm{H}_{23} \mathrm{~N}_{2} \mathrm{O}^{+}[\mathrm{M}+\mathrm{H}]^{+}$ 307.1800 , found 307.1788 .

$N$-(tert-Butyl)-2-(3,4,5-trimethoxyphenyl)quinolin-4-amine (9j). Synthesized in accordance with general procedure 1 . Isolated as a yellow solid $(0.319 \mathrm{~g}, 87 \%): R_{f}=0.10\left(\mathrm{cHex} / \mathrm{EtOAc} / \mathrm{Et}_{3} \mathrm{~N}\right.$ 4:1:0.01); mp 67-69 ${ }^{\circ} \mathrm{C}$; ${ }^{1} \mathrm{H}$ NMR $\left(500.23 \mathrm{MHz}, \mathrm{CDCl}_{3}\right) \delta 8.06(\mathrm{~d}, J=8.5 \mathrm{~Hz}$, $1 \mathrm{H}), 7.68(\mathrm{~d}, J=8.5 \mathrm{~Hz}, 1 \mathrm{H}), 7.63(\mathrm{t}, J=7.5 \mathrm{~Hz}, 1 \mathrm{H}), 7.40(\mathrm{t}, J=7.5$ $\mathrm{Hz}, 1 \mathrm{H}), 7.26$ (s, 2H), 7.03 (s, 1H), 5.06 (bs, 1H), $3.99(\mathrm{~s}, 6 \mathrm{H}), 3.90$ $(\mathrm{s}, 3 \mathrm{H}), 1.59(\mathrm{~s}, 9 \mathrm{H}) ;{ }^{13} \mathrm{C} \mathrm{NMR}\left(125.97 \mathrm{MHz}, \mathrm{CDCl}_{3}\right) \delta 157.8$, 153.3, 184.6, 148.6, 148.4, 138.9, 130.3, 129.1, 124.4, 118.9, 118.4, $104.9,99.1,60.3,56.2,51.5,29.4$; HRMS (ESI) $\mathrm{m} / \mathrm{z}$ calcd for $\mathrm{C}_{22} \mathrm{H}_{27} \mathrm{~N}_{2} \mathrm{O}_{3}{ }^{+}[\mathrm{M}+\mathrm{H}]^{+}$367.2010, found 367.2026.

$\mathrm{N}$-(tert-Butyl)-7-chloro-2-(3,4,5-trimethoxyphenyl)quinolin-4amine (9k). Synthesized in accordance with general procedure 1. Isolated as a yellow solid $(0.328 \mathrm{~g}, 82 \%): R_{f}=0.28(\mathrm{cHex} / \mathrm{EtOAc} /$ $\mathrm{Et}_{3} \mathrm{~N}$ 4:1:0.01); mp 68-70 ${ }^{\circ} \mathrm{C}$; ${ }^{1} \mathrm{H}$ NMR (500.23 $\left.\mathrm{MHz}, \mathrm{CDCl}_{3}\right) \delta$ $8.03(\mathrm{~s}, 1 \mathrm{H}), 7.60(\mathrm{dd}, J=2.0,9.0 \mathrm{~Hz}, 1 \mathrm{H}), 7.33(\mathrm{dt}, J=2.0,9.0 \mathrm{~Hz}$, $1 \mathrm{H}), 7.25(\mathrm{~d}, J=1.5 \mathrm{~Hz}, 2 \mathrm{H}), 7.02(\mathrm{~d}, J=1.5 \mathrm{~Hz}, 1 \mathrm{H}), 5.00(\mathrm{bs}, 1 \mathrm{H})$, $3.98(\mathrm{~s}, 6 \mathrm{H}), 3.91(\mathrm{~s}, 3 \mathrm{H}), 1.59(\mathrm{~s}, 9 \mathrm{H}) ;{ }^{13} \mathrm{C}$ NMR $(125.97 \mathrm{MHz}$, $\left.\mathrm{CDCl}_{3}\right) \delta 158.7,153.3,149.5,148.4,139.1,136.6,134.9,129.2,124.9$, $120.5,116.9,104.8,99.2,60.9,56.3,51.7,29.3$; HRMS (ESI) $\mathrm{m} / z$ calcd for $\mathrm{C}_{22} \mathrm{H}_{26} \mathrm{~N}_{2} \mathrm{O}_{3} \mathrm{Cl}^{+}[\mathrm{M}+\mathrm{H}]^{+}$401.1621, found 401.1634.

Methyl 4-(tert-Butylamino)-2-phenylquinoline-6-carboxylate (9l). Synthesized in accordance with general procedure 1 . Isolated as a yellow solid $(0.147 \mathrm{~g}, 44 \%): R_{f}=0.38\left(\mathrm{cHex} / \mathrm{EtOAc} / \mathrm{Et}_{3} \mathrm{~N}\right.$ 4:1:0.01); mp 131-134 ${ }^{\circ} \mathrm{C}$; ${ }^{1} \mathrm{H}$ NMR (500.23 MHz, $\left.\mathrm{CDCl}_{3}\right) \delta 8.49$ (s, $1 \mathrm{H}), 8.19$ (dd, $J=2.0,8.5 \mathrm{~Hz}, 1 \mathrm{H}), 8.11-8.02(\mathrm{~m}, 3 \mathrm{H}), 7.56-$ $7.49(\mathrm{~m}, 2 \mathrm{H}), 7.49-7.45(\mathrm{~m}, 1 \mathrm{H}), 7.15(\mathrm{~s}, 1 \mathrm{H}), 5.27(\mathrm{bs}, 1 \mathrm{H}), 3.99$ $(\mathrm{s}, 3 \mathrm{H}), 1.62(\mathrm{~s}, 9 \mathrm{H}) ;{ }^{13} \mathrm{C} \mathrm{NMR}\left(125.97 \mathrm{MHz}, \mathrm{CDCl}_{3}\right) \delta 167.0$, $159.8,151.3,149.4,140.7,130.5,129.3,128.7,128.6,127.6$, 125.2, $122.5,117.7,99.5,52.3,51.9,29.3$; HRMS (ESI) $\mathrm{m} / z$ calcd for $\mathrm{C}_{21} \mathrm{H}_{23} \mathrm{~N}_{2} \mathrm{O}_{2}^{+}[\mathrm{M}+\mathrm{H}]^{+}$335.1749, found 335.1759.

4-(4-(tert-Butylamino)quinolin-2-yl)benzonitrile $(9 \mathrm{~m})$. Synthesized in accordance with general procedure 1. Isolated as a yellow solid (0.090 g, 31\%): $R_{f}=0.33\left(\mathrm{cHex} / \mathrm{EtOAc} / \mathrm{Et}_{3} \mathrm{~N} 4: 1: 0.01\right) ; \mathrm{mp}$ $79-81{ }^{\circ} \mathrm{C}$; ${ }^{1} \mathrm{H}$ NMR $\left(500.23 \mathrm{MHz}, \mathrm{CDCl}_{3}\right) \delta 8.18(\mathrm{~d}, J=8.5 \mathrm{~Hz}$, $2 \mathrm{H}), 8.04$ (dd, $J=1.5,8.5 \mathrm{~Hz}, 1 \mathrm{H}), 7.79(\mathrm{~d}, J=8.5 \mathrm{~Hz}, 2 \mathrm{H}), 7.70$ (dd, $J=1.5,8.5 \mathrm{~Hz}, 1 \mathrm{H}), 7.66$ (ddd, $J=1.5,7.0,8.5 \mathrm{~Hz}, 1 \mathrm{H}), 7.45$ (ddd, $J=1.5,7.0,8.5 \mathrm{~Hz}, 1 \mathrm{H}), 7.09(\mathrm{~s}, 1 \mathrm{H}), 5.13(\mathrm{bs}, 1 \mathrm{H}), 1.61(\mathrm{~s}$, $9 \mathrm{H}) ;{ }^{13} \mathrm{C}$ NMR $\left(125.97 \mathrm{MHz}, \mathrm{CDCl}_{3}\right) \delta 155.6,148.7,145.4,132.5$, 130.6, 129.4, 128.1, 125.1, 119.0, 118.9, 118.6, 112.2, 98.7, 51.7, 29.3; HRMS (ESI) $m / z$ calcd for $\mathrm{C}_{20} \mathrm{H}_{20} \mathrm{~N}_{3}{ }^{+}[\mathrm{M}+\mathrm{H}]^{+} 302.1648$, found 302.1656.

$N$-(tert-Butyl)-2-butylquinolin-4-amine (9n). Synthesized in accordance with general procedure 1 . Isolated as a brown solid $(0.129 \mathrm{~g}$, 
50\%): $R_{f}=0.53\left(\mathrm{EtOAc} / \mathrm{Et}_{3} \mathrm{~N}\right.$ 100:1); $\mathrm{mp} \mathrm{68-70}{ }^{\circ} \mathrm{C} ;{ }^{1} \mathrm{H} \mathrm{NMR}$ $\left(500.23 \mathrm{MHz}, \mathrm{CDCl}_{3}\right) \delta 7.91(\mathrm{~d}, J=8.5 \mathrm{~Hz}, 1 \mathrm{H}), 7.62(\mathrm{~d}, J=8.5 \mathrm{~Hz}$, $1 \mathrm{H}), 7.59-7.53(\mathrm{~m}, 1 \mathrm{H}), 7.35$ (ddd, $J=1.0,7.0,8.5 \mathrm{~Hz}, 1 \mathrm{H}), 6.57(\mathrm{~s}$, $1 \mathrm{H}), 4.91(\mathrm{bs}, 1 \mathrm{H}), 2.87-2.79(\mathrm{~m}, 2 \mathrm{H}), 1.81-1.72(\mathrm{~m}, 2 \mathrm{H}), 1.54(\mathrm{~s}$, $9 \mathrm{H}), 1.51-1.40(\mathrm{~m}, 2 \mathrm{H}), 0.96(\mathrm{t}, J=7.5 \mathrm{~Hz}, 3 \mathrm{H}) ;{ }^{13} \mathrm{C}$ NMR $(125.97$ $\left.\mathrm{MHz} \mathrm{CDCl}_{3}\right) \delta 163.0,148.4,147.8,129.5,128.7,123.7,118.8,118.1$, $100.8,51.4,39.6,32.4,29.3,22.7,14.1$; HRMS (ESI) $\mathrm{m} / z$ calcd for $\mathrm{C}_{17} \mathrm{H}_{25} \mathrm{~N}_{2}^{+}[\mathrm{M}+\mathrm{H}]^{+}$257.2007, found 257.2023.

$\mathrm{N}$-(tert-Butyl)-2-decylquinolin-4-amine (9o). Synthesized in accordance with general procedure 1 . Isolated as a red-brown wax $(0.255 \mathrm{~g}, 75 \%): R_{f}=0.62$ (EtOAc:Et ${ }_{3} \mathrm{~N} \mathrm{100:1);}{ }^{1} \mathrm{H}$ NMR (500.23 $\left.\mathrm{MHz}_{\mathrm{CDCl}}\right) \delta 7.92(\mathrm{~d}, J=8.5 \mathrm{~Hz}, 1 \mathrm{H}), 7.62(\mathrm{~d}, J=8.5 \mathrm{~Hz}, 1 \mathrm{H})$, $7.56(\mathrm{ddd}, J=1.5,7.0,8.5 \mathrm{~Hz}, 1 \mathrm{H}), 7.34(\mathrm{t}, J=1.5,7.5,7.0 \mathrm{~Hz}, 1 \mathrm{H})$, $6.57(\mathrm{~s}, 1 \mathrm{H}), 4.91(\mathrm{bs}, 1 \mathrm{H}), 2.82(\mathrm{t}, J=8.0 \mathrm{~Hz}, 2 \mathrm{H}), 1.78(\mathrm{p}, J=8.0$ $\mathrm{Hz}, 2 \mathrm{H}), 1.53(\mathrm{~s}, 9 \mathrm{H}), 1.46-1.38(\mathrm{~m}, 2 \mathrm{H}), 1.38-1.32(\mathrm{~m}, 2 \mathrm{H})$, $1.31-1.21(\mathrm{~m}, 10 \mathrm{H}), 0.87(\mathrm{t}, J=7.0 \mathrm{~Hz}, 3 \mathrm{H}) ;{ }^{13} \mathrm{C}$ NMR $(125.97$ $\left.\mathrm{MHz}, \mathrm{CDCl}_{3}\right) \delta 163.0,148.4,147.8,129.5,128.6,123.7,118.8,118.1$, $100.8,51.3,39.9,31.9,30.2,29.62,29.58,29.56,29.3,29.2,22.6,14.1$; HRMS (ESI) $m / z$ calcd for $\mathrm{C}_{23} \mathrm{H}_{37} \mathrm{~N}_{2}{ }^{+}[\mathrm{M}+\mathrm{H}]^{+} 341.2943$, found 341.2965.

$\mathrm{N}$-Isopropyl-2-phenylquinolin-4-amine (9p). Synthesized in accordance with general procedure 2 . Isolated as a red-brown solid

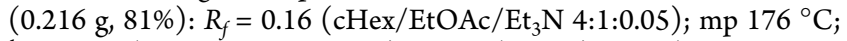
${ }^{1} \mathrm{H} \mathrm{NMR}\left(500.23 \mathrm{MHz}, \mathrm{CDCl}_{3}\right) \delta 8.12(\mathrm{~m}, 3 \mathrm{H}), 7.72(\mathrm{~d}, J=8.5 \mathrm{~Hz}$, $1 \mathrm{H}), 7.68-7.61(\mathrm{~m}, 1 \mathrm{H}), 7.51(\mathrm{t}, J=7.5 \mathrm{~Hz}, 2 \mathrm{H}), 7.48-7.38(\mathrm{~m}$, $2 \mathrm{H}), 6.89(\mathrm{~s}, 1 \mathrm{H}), 4.87(\mathrm{bd}, J=7.0 \mathrm{~Hz}, 1 \mathrm{H}), 4.04-3.92(\mathrm{~m}, 1 \mathrm{H})$, $1.40(\mathrm{dd}, J=1.5,6.5 \mathrm{~Hz}, 6 \mathrm{H}) ;{ }^{13} \mathrm{C}$ NMR $\left(125.97 \mathrm{MHz}, \mathrm{CDCl}_{3}\right) \delta$ $158.4,149.0,148.7,141.1,130.3,129.1,128.7,128.5,127.5,124.2$, 119.0, 117.8, 97.0, 43.9, 22.5; HRMS (ESI) $m / z$ calcd for $\mathrm{C}_{18} \mathrm{H}_{19} \mathrm{~N}_{2}{ }^{+}$ $[\mathrm{M}+\mathrm{H}]^{+}$263.1539, found 263.1556 .

$\mathrm{N}$-Cyclohexyl-6-fluoro-2-phenylquinolin-4-amine (9q). Synthesized in accordance with general procedure 2. Isolated as a yellow solid $(0.229 \mathrm{~g}, 80 \%): R_{f}=0.50\left(\mathrm{cHex} / \mathrm{EtOAc} / \mathrm{Et}_{3} \mathrm{~N} 4: 1: 0.01\right) ; \mathrm{mp}$ 146-149 ${ }^{\circ} \mathrm{C} ;{ }^{1} \mathrm{H}$ NMR $\left(500.23 \mathrm{MHz}, \mathrm{CDCl}_{3}\right) \delta 8.09-8.06(\mathrm{~m}, 1 \mathrm{H})$, $8.04(\mathrm{~d}, J=8.0 \mathrm{~Hz}, 2 \mathrm{H}), 7.51(\mathrm{~d}, J=7.0 \mathrm{~Hz}, 2 \mathrm{H}), 7.47-7.37(\mathrm{~m}$, $2 \mathrm{H}), 7.34(\mathrm{dd}, J=2.0,10.0 \mathrm{~Hz}, 1 \mathrm{H}), 6.86(\mathrm{~s}, 1 \mathrm{H}), 4.71(\mathrm{bd}, J=7.5$ $\mathrm{Hz}, 1 \mathrm{H}), 3.66-3.56(\mathrm{~m}, 1 \mathrm{H}), 2.20(\mathrm{dd}, J=4.0,13.0 \mathrm{~Hz}, 2 \mathrm{H}), 1.89-$ $1.81(\mathrm{~m}, 2 \mathrm{H}), 1.76-1.70(\mathrm{~m}, 1 \mathrm{H}), 1.53-1.44(\mathrm{~m}, 2 \mathrm{H}), 1.42-1.30$ $(\mathrm{m}, 3 \mathrm{H}) ;{ }^{13} \mathrm{C}$ NMR (125.97 $\left.\mathrm{MHz}, \mathrm{CDCl}_{3}\right) \delta 159.5(\mathrm{~d}, J=245.0)$, $157.9(\mathrm{~d}, J=2.5 \mathrm{~Hz}), 148.6(\mathrm{~d}, J=5.0 \mathrm{~Hz}), 145.7,140.8,132.5(\mathrm{~d}, J=$ $8.5 \mathrm{~Hz}), 128.9,128.6,127.5,118.8(\mathrm{~d}, J=25.0 \mathrm{~Hz}), 118.2(\mathrm{~d}, J=8.0$ $\mathrm{Hz}), 103.4(\mathrm{~d}, J=23.0 \mathrm{~Hz}), 97.3,51.3,32.8,25.7,24.8$; HRMS (ESI) $m / z$ calcd for $\mathrm{C}_{21} \mathrm{H}_{22} \mathrm{~N}_{2} \mathrm{~F}^{+}[\mathrm{M}+\mathrm{H}]^{+} 321.1757$, found 321.1776.

7-Chloro-N-isopropyl-2-phenylquinolin-4-amine (9r). Synthesized in accordance with general procedure 2 . Isolated as a yellow solid (0.175 g, 60\%): $R_{f}=0.47\left(\mathrm{cHex} / \mathrm{EtOAc} / \mathrm{Et}_{3} \mathrm{~N} 4: 1: 0.01\right) ; \mathrm{mp}$ 147-148 ${ }^{\circ} \mathrm{C}$; ${ }^{1} \mathrm{H}$ NMR $\left(500.23 \mathrm{MHz}, \mathrm{CDCl}_{3}\right) \delta 8.11-8.00(\mathrm{~m}, 3 \mathrm{H})$, $7.65(\mathrm{~d}, J=9.0 \mathrm{~Hz}, 1 \mathrm{H}), 7.53-7.48(\mathrm{~m}, 2 \mathrm{H}), 7.47-7.43(\mathrm{~m}, 1 \mathrm{H})$, 7.34 (dd, $J=2.0,9.0 \mathrm{~Hz}, 1 \mathrm{H}), 6.85(\mathrm{~s}, 1 \mathrm{H}), 4.88$ (bs, $1 \mathrm{H}), 4.04-3.91$ $(\mathrm{m}, 1 \mathrm{H}), 1.40(\mathrm{~d}, J=6.5 \mathrm{~Hz}, 6 \mathrm{H}) ;{ }^{13} \mathrm{C} \mathrm{NMR}\left(125.97 \mathrm{MHz}, \mathrm{CDCl}_{3}\right) \delta$ $159.3,149.4,149.2$, 140.5, 135.0, 129.1, 129.0, 128.6, 127.5, 124.9, 120.6, 116.2, 97.2, 44.1, 22.5; HRMS (ESI) $\mathrm{m} / \mathrm{z}$ calcd for $\mathrm{C}_{18} \mathrm{H}_{18} \mathrm{~N}_{2} \mathrm{Cl}^{+}[\mathrm{M}+\mathrm{H}]^{+}$297.1150, found 297.1163.

$\mathrm{N}$-Pentyl-2-phenylquinolin-4-amine (9s). Synthesized in accordance with general procedure 2 . Isolated as a red-brown wax $(0.131 \mathrm{~g}$, 48\%): $R_{f}=0.35\left(\mathrm{cHex} / \mathrm{EtOAC} \mathrm{Et}_{3} \mathrm{~N}\right.$ 4:1:0.01); ${ }^{1} \mathrm{H} \mathrm{NMR}(500.23$ $\left.\mathrm{MHz}, \mathrm{CDCl}_{3}\right) \delta 8.14-8.06(\mathrm{~m}, 3 \mathrm{H}), 7.72(\mathrm{~d}, J=8.5 \mathrm{~Hz}, 1 \mathrm{H}), 7.65(\mathrm{t}$, $J=7.5 \mathrm{~Hz}, 1 \mathrm{H}), 7.51(\mathrm{t}, J=7.5 \mathrm{~Hz}, 2 \mathrm{H}), 7.47-7.38(\mathrm{~m}, 2 \mathrm{H}), 6.86(\mathrm{~s}$, $1 \mathrm{H}), 5.00(\mathrm{bt}, J=5.0 \mathrm{~Hz}, 1 \mathrm{H}), 3.36(\mathrm{q}, J=6.5 \mathrm{~Hz}, 2 \mathrm{H}), 1.79(\mathrm{p}, J=$ $7.5 \mathrm{~Hz}, 2 \mathrm{H}), 1.53-1.37(\mathrm{~m}, 4 \mathrm{H}), 0.96(\mathrm{t}, J=7.0 \mathrm{~Hz}, 3 \mathrm{H}) ;{ }^{13} \mathrm{C} \mathrm{NMR}$ $\left(125.97 \mathrm{MHz}, \mathrm{CDCl}_{3}\right) \delta 158.4,150.1,148.6,141.0,130.2,129.1$, 128.8, 128.5, 127.5, 124.3, 119.0, 117.8, 96.6, 43.2, 29.3 28.6, 22.4, 14.0; HRMS (ESI) $m / z$ calcd for $\mathrm{C}_{20} \mathrm{H}_{23} \mathrm{~N}_{2}{ }^{+}[\mathrm{M}+\mathrm{H}]^{+}$291.1851, found 291.1846 .

$\mathrm{N}$-Isopropyl-2-(4-(trifluoromethyl)phenyl)quinolin-4-amine (9t). Synthesized in accordance with general procedure 2 . Isolated as a

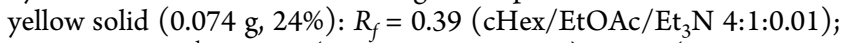
mp 60-63 ${ }^{\circ} \mathrm{C} ;{ }^{1} \mathrm{H}$ NMR $\left(500.23 \mathrm{MHz}, \mathrm{CDCl}_{3}\right) \delta 8.18(\mathrm{~d}, J=8.0 \mathrm{~Hz}$, $2 \mathrm{H}), 8.07(\mathrm{~d}, J=8.5 \mathrm{~Hz}, 1 \mathrm{H}), 7.79-7.70(\mathrm{~m}, 3 \mathrm{H}), 7.67(\mathrm{t}, J=7.5 \mathrm{~Hz}$, $1 \mathrm{H}), 7.44(\mathrm{t}, J=7.5 \mathrm{~Hz}, 1 \mathrm{H}), 6.86(\mathrm{~s}, 1 \mathrm{H}), 4.93(\mathrm{bd}, J=7.0 \mathrm{~Hz}, 1 \mathrm{H})$,
4.05-3.96 (m, 1H), $1.41(\mathrm{~d}, J=6.5 \mathrm{~Hz}, 6 \mathrm{H}) ;{ }^{13} \mathrm{C}$ NMR $(125.97$ $\left.\mathrm{MHz}, \mathrm{CDCl}_{3}\right) \delta 156.8,149.3,148.7,144.4,130.6(\mathrm{q}, J=32.5 \mathrm{~Hz})$, $130.4,129.4,127.8,125.5(\mathrm{q}, J=4.0 \mathrm{~Hz}), 124.8,124.3(\mathrm{q}, J=271.5$ $\mathrm{Hz}$ ), 119.0, 117.9, 96.8, 40.0, 22.5; HRMS (ESI) $\mathrm{m} / z$ calcd for $\mathrm{C}_{19} \mathrm{H}_{18} \mathrm{~N}_{2} \mathrm{~F}_{3}^{+}[\mathrm{M}+\mathrm{H}]^{+}$331.1413, found 331.1429.

Methyl 4-(Cyclohexylamino)-2-(3,4,5-trimethoxyphenyl)quinoline-6-carboxylate (9u). Synthesized in accordance with general procedure 2 . Isolated as a dark yellow solid (0.167 g, 39\%): $R_{f}=0.13$ (cHex/EtOAc/ $/ \mathrm{Et}_{3} \mathrm{~N}$ 4:1:0.01); mp 141-143 ${ }^{\circ} \mathrm{C} ;{ }^{1} \mathrm{H}$ NMR $\left(500.23 \mathrm{MHz}, \mathrm{CDCl}_{3}\right) \delta 8.52(\mathrm{~d}, J=2.0 \mathrm{~Hz}, 1 \mathrm{H}), 8.20(\mathrm{dd}, J=2.0$, $9.0 \mathrm{~Hz}, 1 \mathrm{H}), 8.03(\mathrm{~d}, J=9.0 \mathrm{~Hz}, 1 \mathrm{H}), 7.27(\mathrm{~s}, 2 \mathrm{H}), 6.80(\mathrm{~s}, 1 \mathrm{H}), 5.19$ (bd, $J=7.0 \mathrm{~Hz}), 4.11-3.95(\mathrm{~m}, 9 \mathrm{H}), 3.91(\mathrm{~s}, 3 \mathrm{H}), 3.63(\mathrm{dtt}, J=3.5$, 7.0, $10.0 \mathrm{~Hz}, 1 \mathrm{H}), 2.24-2.17(\mathrm{~m}, 2 \mathrm{H}), 1.97-1.82(\mathrm{~m}, 2 \mathrm{H}), 1.81-$ $1.68(\mathrm{~m}, 2 \mathrm{H}), 1.52-1.37(\mathrm{~m}, 4 \mathrm{H}) ;{ }^{13} \mathrm{C} \mathrm{NMR}\left(125.97 \mathrm{MHz}, \mathrm{CDCl}_{3}\right)$ $\delta 167.0,160.3,153.3,151.1,149.9,139.3,136.5,130.2,128.8,125.1$, $122.7,117.0,105.1,97.1,60.9,56.3,52.3,51.5,32.7,25.6,24.9$; HRMS (ESI) $m / z$ calcd for $\mathrm{C}_{26} \mathrm{H}_{31} \mathrm{~N}_{2} \mathrm{O}_{5}{ }^{+}[\mathrm{M}+\mathrm{H}]^{+} 451.2220$, found 451.2236.

$N^{1}, N^{1}$-Diethyl- $N^{4}$-(2-phenylquinolin-4-yl)pentane-1,4-diamine $(9 w)$. Synthesized in accordance with general procedure 2. Isolated as a red-brown wax $(0.287 \mathrm{~g}, 79 \%): R_{f}=0.09\left(\mathrm{EtOAc} / \mathrm{Et}_{3} \mathrm{~N} \mathrm{100:1)} ;{ }^{1} \mathrm{H}\right.$ NMR (500.23 MHz, $\left.\mathrm{CDCl}_{3}\right) \delta 8.09-8.02(\mathrm{~m}, 3 \mathrm{H}), 7.76(\mathrm{~d}, J=8.0$ $\mathrm{Hz}, 1 \mathrm{H}), 7.67-7.60(\mathrm{~m}, 1 \mathrm{H}), 7.50(\mathrm{t}, J=7.5 \mathrm{~Hz}, 2 \mathrm{H}), 7.47-7.37(\mathrm{~m}$, $2 \mathrm{H}), 6.86(\mathrm{~s}, 1 \mathrm{H}), 5.18(\mathrm{bd}, J=7.5 \mathrm{~Hz}, 1 \mathrm{H}), 3.90-3.80(\mathrm{~m}, 1 \mathrm{H})$, $2.57(\mathrm{q}, J=7.0 \mathrm{~Hz}, 4 \mathrm{H}), 2.50(\mathrm{t}, J=7.0 \mathrm{~Hz}, 2 \mathrm{H}), 1.85-1.75(\mathrm{~m}, 1 \mathrm{H})$, $1.73-1.63(\mathrm{~m}, 2 \mathrm{H}), 1.37(\mathrm{~d}, J=6.5 \mathrm{H}, 3 \mathrm{H}), 1.03(\mathrm{t}, J=7.0 \mathrm{~Hz}, 6 \mathrm{H})$; ${ }^{13} \mathrm{C}$ NMR (125.97 MHz, $\left.\mathrm{CDCl}_{3}\right) \delta 158.5,149.3,148.8,141.2,130.3$, $129.1,128.7,128.6,127.5,124.2,119.2,117.9,96.9,52.6,48.2,46.8$, 34.7, 23.8, 20.4, 11.4; HRMS (ESI) $m / z$ calcd for $\mathrm{C}_{24} \mathrm{H}_{32} \mathrm{~N}_{3}{ }^{+}[\mathrm{M}+$ $\mathrm{H}]^{+} 362.2584$, found 362.2595 .

$N^{4}$-(3-(Benzo[d][1,3]dioxol-5-yl)-6-chloronaphthalen-1-yl)- $N^{1}, N^{1}$ diethylpentane-1,4-diamine (9x). Synthesized in accordance with general procedure 2 . Isolated as a yellow-brown solid $(0.327 \mathrm{~g}, 78 \%)$ : $R_{f}=0.15\left(\mathrm{EtOAc} / \mathrm{Et}_{3} \mathrm{~N}\right.$ 99:1); mp $48-50{ }^{\circ} \mathrm{C}$; ${ }^{1} \mathrm{H}$ NMR (500.23 $\left.\mathrm{MHz}, \mathrm{CDCl}_{3}\right) \delta 7.98(\mathrm{~d}, J=2.0 \mathrm{~Hz}, 1 \mathrm{H}), 7.63(\mathrm{~d}, J=9.0 \mathrm{~Hz}, 1 \mathrm{H})$, $7.60(\mathrm{~d}, J=2.0 \mathrm{~Hz}, 1 \mathrm{H}), 7.56(\mathrm{~d}, J=8.0 \mathrm{~Hz}, 1 \mathrm{H}), 7.29(\mathrm{~d}, J=9.0 \mathrm{~Hz}$, $1 \mathrm{H}), 6.91(\mathrm{~d}, J=8.0 \mathrm{~Hz}, 1 \mathrm{H}), 6.76(\mathrm{~s}, 1 \mathrm{H}), 6.01(\mathrm{~s}, 2 \mathrm{H}), 5.25(\mathrm{bd}, J=$ $7.0 \mathrm{~Hz}, 1 \mathrm{H}), 3.79$ (hept, $J=6.5 \mathrm{~Hz}, 1 \mathrm{H}), 2.53(\mathrm{q}, J=7.0 \mathrm{~Hz}, 4 \mathrm{H})$, $2.46(\mathrm{t}, J=7.0 \mathrm{~Hz}, 2 \mathrm{H}), 1.81-1.65(\mathrm{~m}, 2 \mathrm{H}), 1.65-1.59(\mathrm{~m}, 2 \mathrm{H})$, $1.34(\mathrm{~d}, J=6.5 \mathrm{~Hz}, 3 \mathrm{H}), 1.00(\mathrm{t}, J=7.0 \mathrm{~Hz}, 6 \mathrm{H}) ;{ }^{13} \mathrm{C} \operatorname{NMR}(125.97$ $\left.\mathrm{MHz}, \mathrm{CDCl}_{3}\right) \delta 158.6,149.5,149.3,148.5,148.0,135.1,134.8,128.9$, $124.4,121.5,120.9,116.3,108.2,107.9,101.2,96.5,52.4,48.2,46.7$, 34.5, 23.8, 20.2, 11.3; HRMS (ESI) $m / z$ calcd for $\mathrm{C}_{25} \mathrm{H}_{31} \mathrm{~N}_{3} \mathrm{O}_{2} \mathrm{Cl}^{+}[\mathrm{M}$ $+\mathrm{H}]^{+} 440.2093$, found 440.2092 .

$N^{4}$-(7-Chloro-2-phenylquinolin-4-yl)- $N^{1}, N^{1}$-diethylpentane-1,4diamine (9y). Synthesized in accordance with general procedure 2. Isolated as a red-brown wax $(0.326 \mathrm{~g}, 82 \%): R_{f}=0.14\left(\mathrm{EtOAc} / \mathrm{Et}_{3} \mathrm{~N}\right.$ 99:1); ${ }^{1} \mathrm{H}$ NMR (500.23 MHz, $\left.\mathrm{CDCl}_{3}\right) \delta 8.08-8.02(\mathrm{~m}, 3 \mathrm{H}), 7.67$ $(\mathrm{d}, J=9.0 \mathrm{~Hz}, 1 \mathrm{H}), 7.50(\mathrm{t}, J=7.0 \mathrm{~Hz}, 2 \mathrm{H}), 7.48-7.40(\mathrm{~m}, 1 \mathrm{H}), 7.33$ (dd, $J=2.0,9.0 \mathrm{~Hz}, 1 \mathrm{H}), 6.85(\mathrm{~s}, 1 \mathrm{H}), 5.29(\mathrm{bd}, J=7.0 \mathrm{~Hz}, 1 \mathrm{H}), 3.82$ (hept, $J=6.5,1 \mathrm{H}), 2.54(\mathrm{q}, J=7.0 \mathrm{~Hz}, 4 \mathrm{H}), 2.47(\mathrm{t}, J=7.0 \mathrm{~Hz}, 2 \mathrm{H})$, $1.83-1.74(\mathrm{~m}, 1 \mathrm{H}), 1.74-1.60(\mathrm{~m}, 3 \mathrm{H}), 1.36(\mathrm{~d}, J=6.5 \mathrm{~Hz}, 3 \mathrm{H})$, $1.01(\mathrm{t}, J=7.0 \mathrm{~Hz}, 6 \mathrm{H}) ;{ }^{13} \mathrm{C}$ NMR $\left(125.97 \mathrm{MHz}, \mathrm{CDCl}_{3}\right) \delta 159.4$, $149.6,149.5,140.7,134.9,129.1,129.0,128.6,127.5,124.7,120.9$, $116.4,97.1,52.5,48.3,46.8,34.6,23.8,20.3,11.3$; HRMS (ESI) $m / z$ calcd for $\mathrm{C}_{24} \mathrm{H}_{31} \mathrm{~N}_{3} \mathrm{Cl}^{+}[\mathrm{M}+\mathrm{H}]^{+}$396.2195, found 396.2213.

$N^{4}$-(2-Cyclohexyl-7-fluoroquinolin-4-yl)- $N^{1}, N^{1}$-diethylpentane1,4-diamine (9z). Synthesized in accordance with general procedure 2. Isolated as a red-brown wax $(0.268 \mathrm{~g}, 70 \%): R_{f}=0.14$ (EtOAc/ $\mathrm{Et}_{3} \mathrm{~N}$ 99:1); ${ }^{1} \mathrm{H}$ NMR (500.23 MHz, $\left.\mathrm{CDCl}_{3}\right) \delta 7.71-7.64(\mathrm{~m}, 1 \mathrm{H})$, $7.53(\mathrm{dt}, J=2.0,10.5 \mathrm{~Hz}, 1 \mathrm{H}), 7.06(\mathrm{t}, J=8.5 \mathrm{~Hz}, 1 \mathrm{H}), 6.28(\mathrm{~d}, J=$ $2.0 \mathrm{~Hz}, 1 \mathrm{H}), 5.16(\mathrm{bd}, J=7.0 \mathrm{~Hz}, 1 \mathrm{H}), 3.72(\mathrm{p}, J=6.5 \mathrm{~Hz}, 1 \mathrm{H}), 2.72$ $(\mathrm{td}, J=3.0,12.0 \mathrm{~Hz}, 1 \mathrm{H}), 2.52(\mathrm{q}, J=7.0 \mathrm{~Hz}, 4 \mathrm{H}), 2.45(\mathrm{t}, J=7.0 \mathrm{~Hz}$, $2 \mathrm{H}), 2.01-1.94(\mathrm{~m}, 2 \mathrm{H}), 1.87-1.81(\mathrm{~m}, 2 \mathrm{H}), 1.77-1.69(\mathrm{~m}, 2 \mathrm{H})$, $1.65-1.50(\mathrm{~m}, 5 \mathrm{H}), 1.42(\mathrm{q}, J=11.5 \mathrm{~Hz}, 2 \mathrm{H}), 1.34-1.24(\mathrm{~m}, 1 \mathrm{H})$, $1.30(\mathrm{~d}, J=6.5 \mathrm{~Hz}, 3 \mathrm{H}), 1.00(\mathrm{td}, J=2.0,7.0,6 \mathrm{H}) ;{ }^{13} \mathrm{C} \mathrm{NMR}$ $\left(125.97 \mathrm{MHz}, \mathrm{CDCl}_{3}\right) \delta 168.7,162.9(\mathrm{~d}, J=247.5 \mathrm{~Hz}), 149.6(\mathrm{~d}, J=$ $12.5 \mathrm{~Hz}), 149.3,121.5$ (d, $J=10.5 \mathrm{~Hz}), 114.8,113.1(\mathrm{~d}, J=25.0 \mathrm{~Hz})$, $112.8(\mathrm{~d}, J=20.0 \mathrm{~Hz}), 96.3,52.5,48.0,47.9,46.7,34.5,32.89,32.86$, 
26.5, 26.0, 23.7, 20.2, 11.2; HRMS (ESI) $m / z$ calcd for $\mathrm{C}_{24} \mathrm{H}_{37} \mathrm{~N}_{3} \mathrm{~F}^{+}$ $[\mathrm{M}+\mathrm{H}]^{+}$386.2958, found 386.2970.

$\mathrm{N}$-(6-Methylheptan-2-yl)-2-(naphthalen-2-yl)quinolin-4-amine (9aa). Synthesized in accordance with general procedure 2. Isolated as a red-brown solid $(0.294 \mathrm{~g}, 77 \%): R_{f}=0.26\left(\mathrm{cHex} / \mathrm{EtOAc} / \mathrm{Et}_{3} \mathrm{~N}\right.$ 4:1:0.01); mp 128-129 ${ }^{\circ} \mathrm{C}$; ${ }^{1} \mathrm{H}$ NMR $\left(500.23 \mathrm{MHz}, \mathrm{CDCl}_{3}\right) \delta 8.57-$ $8.53(\mathrm{~m}, 1 \mathrm{H}), 8.26(\mathrm{dd}, J=2.8,8.5 \mathrm{~Hz}, 1 \mathrm{H}), 8.13(\mathrm{~d}, J=8.5 \mathrm{~Hz}, 1 \mathrm{H})$, $8.01-7.98 \mathrm{~m}, 1 \mathrm{H}), 7.98(\mathrm{~d}, J=9.0 \mathrm{~Hz}, 1 \mathrm{H}), 7.92-7.87(\mathrm{~m}, 1 \mathrm{H})$, $7.76-7.72(\mathrm{~m}, 1 \mathrm{H}), 7.67(\mathrm{ddd}, J=1.5,7.0,8.5 \mathrm{~Hz}, 1 \mathrm{H}), 7.54-7.49$ (m, 2H), 7.44 (ddd, $J=1.5,7.0,8.5 \mathrm{~Hz}, 1 \mathrm{M}), 7.01(\mathrm{~s}, 1 \mathrm{H}), 4.90$ (bd, $J$ $=7.5 \mathrm{~Hz}, 1 \mathrm{H}), 3.89$ (hept, $J=6.5 \mathrm{~Hz}, 1 \mathrm{H}), 1.81-1.73(\mathrm{~m}, 1 \mathrm{H})$, $1.70-1.53(\mathrm{~m}, 3 \mathrm{H}), 1.53-1.44(\mathrm{~m}, 2 \mathrm{H}), 1.39(\mathrm{~d}, J=6.5 \mathrm{~Hz}, 3 \mathrm{H})$, $1.30-1.24(\mathrm{~m}, 2 \mathrm{H}), 0.90(\mathrm{~d}, J=1.5 \mathrm{~Hz}, 3 \mathrm{H}), 0.89(\mathrm{~d}, J=1.5 \mathrm{~Hz}$, $3 \mathrm{H}) ;{ }^{13} \mathrm{C}$ NMR $\left(125.97 \mathrm{MHz}, \mathrm{CDCl}_{3}\right) \delta 158.2,149.4,148.8,138.3$, $133.7,133.4,130.3,129.3,128.7,128.2,127.7,126.9,126.4,126.1$, $125.4,124.4,119.0,117.9,97.0,48.3,38.9,37.1,27.9,24.0,22.60$, 22.58, 20.4; HRMS (ESI) $m / z$ calcd for $\mathrm{C}_{27} \mathrm{H}_{31} \mathrm{~N}_{2}{ }^{+}[\mathrm{M}+\mathrm{H}]^{+}$ 383.2475, found 383.2490.

Ethyl 4-((7-Chloro-2-phenylquinolin-4-yl)amino)piperi-dine-1carboxylate (9ab). Synthesized in accordance with general procedure 2. Isolated as a yellow-orange solid $(0.277 \mathrm{~g}, 68 \%): R_{f}=0.11(\mathrm{cHex} /$ $\mathrm{EtOAc} / \mathrm{Et}_{3} \mathrm{~N}$ 4:1:0.01); mp $186-187{ }^{\circ} \mathrm{C}$; ${ }^{1} \mathrm{H}$ NMR (500.23 MHz, $\left.\mathrm{CDCl}_{3}\right) \delta 8.07(\mathrm{~d}, J=2.0 \mathrm{~Hz}, 1 \mathrm{H}), 8.05-8.03(\mathrm{~m}, 1 \mathrm{H}), 8.03-8.01$ $(\mathrm{m}, 1 \mathrm{H}), 7.65 \mathrm{~d}, J=9.0 \mathrm{~Hz}, 1 \mathrm{H}), 7.68-7.62(\mathrm{~m}, 2 \mathrm{H}), 7.50-7.42(\mathrm{~m}$, $1 \mathrm{H}), 7.35(\mathrm{dd}, J=2.0,9.0 \mathrm{~Hz}, 1 \mathrm{H}), 6.85(\mathrm{~s}, 1 \mathrm{H}), 4.95$ (bs, $1 \mathrm{H})$, $4.32-4.25 \mathrm{~m}, 2 \mathrm{H}), 4.17(\mathrm{q}, J=7.0 \mathrm{~Hz}, 2 \mathrm{H}), 3.80(\mathrm{tdt}, J=4.0,7.5$, $10.5 \mathrm{~Hz}, 1 \mathrm{H}), 3.08(\mathrm{t}, J=12.5 \mathrm{~Hz}, 2 \mathrm{H}), 2.28-2.14(\mathrm{~m}, 2 \mathrm{H}), 1.64-$ $1.48(\mathrm{~m}, 2 \mathrm{H}), 1.29(\mathrm{t}, J=7.0 \mathrm{~Hz}, 3 \mathrm{H}) ;{ }^{13} \mathrm{C}$ NMR (125.97 MHz, $\left.\mathrm{CDCl}_{3}\right): 159.3,155.4,148.7,140.3,135.3,129.3,129.1,128.7,127.6$, 125.2, 120.6, 116.2, 97.2, 61.6, 49.7, 42.6, 31.7, 14.7; HRMS (ESI) m/ $z$ calcd for $\mathrm{C}_{23} \mathrm{H}_{25} \mathrm{~N}_{3} \mathrm{O}_{2} \mathrm{Cl}^{+}[\mathrm{M}+\mathrm{H}]^{+} 410.1625$, found 410.1630 .

$\mathrm{N}, \mathrm{N}$-Diethyl-4-isocyanopentan-1-amine (7w). A solution of $N^{1}, N^{1}$-diethylpentane-1,4-diamine $(3.87 \mathrm{~mL}, 20 \mathrm{mmol})$ in ethyl formate $(4.82 \mathrm{~mL}, 60 \mathrm{mmol})$ was refluxed for $16 \mathrm{~h}$, after which the solvent was removed in vacuo. To a solution of the crude product $(3.00 \mathrm{~g}, 16.1 \mathrm{mmol})$ in dry $\mathrm{CH}_{2} \mathrm{Cl}_{2}(80 \mathrm{~mL})$ was added DIPEA (7.01 $\mathrm{mL}, 40.25 \mathrm{mmol})$ and cooled to $-40{ }^{\circ} \mathrm{C}$. Triphosgene $(1.67 \mathrm{~g}, 5.64$ $\mathrm{mmol}$ ) was added portionwise, after which the mixture was allowed to warm to $0{ }^{\circ} \mathrm{C}$ and stirred for $2 \mathrm{~h}$. The crude product was poured in water $(80 \mathrm{~mL})$, and the organic layer was dried over $\mathrm{Na}_{2} \mathrm{SO}_{4}$, filtered, and concentrated in vacuo to afford the title compound as a pale yellow liquid $(2.370 \mathrm{~g}, 88 \%): R_{f}=0.11\left(\mathrm{EtOAc} / \mathrm{MeOH} / \mathrm{Et}_{3} \mathrm{~N}\right.$ 9:1:0.09); ${ }^{1} \mathrm{H}$ NMR (500.23 $\left.\mathrm{MHz}, \mathrm{CDCl}_{3}\right) \delta 3.71-3.59(\mathrm{~m}, 1 \mathrm{H})$, $2.52(\mathrm{q}, J=7.0 \mathrm{~Hz}, 4 \mathrm{H}), 2.44(\mathrm{t}, J=7.0 \mathrm{~Hz}, 2 \mathrm{H}), 1.70-1.52(\mathrm{~m}, 4 \mathrm{H})$, $1.37\left(\mathrm{dt}, J_{\mathrm{HN}}=2.0 \mathrm{~Hz}, J_{\mathrm{HH}}=7.0 \mathrm{~Hz}, 3 \mathrm{H}\right), 1.02(\mathrm{t}, J=7.0 \mathrm{~Hz}, 3 \mathrm{H})$; ${ }^{13} \mathrm{C}\left(125.97 \mathrm{MHz}, \mathrm{CDCl}_{3}\right) \delta 154.2\left(\mathrm{t}, J_{\mathrm{C}(\mathrm{sp}) \mathrm{N}}=5.0 \mathrm{~Hz}\right), 52.0,50.3(\mathrm{t}$, $\left.J_{\mathrm{C}(\mathrm{sp} 3) \mathrm{N}}=5.5 \mathrm{~Hz}\right), 46.8,34.8,23.3,21.8,11.6$; HRMS (ESI) $\mathrm{m} / z$ calcd for $\mathrm{C}_{10} \mathrm{H}_{21} \mathrm{~N}_{2}^{+}[\mathrm{M}+\mathrm{H}]^{+}$169.1695, found 169.1705.

Ethyl 4-Isocyanopiperidine-1-carboxylate (7ab). Ethyl 4-aminopiperidine-1-carboxylate $(4.03 \mathrm{~mL}, 29.0 \mathrm{mmol})$ was refluxed in ethyl formate $(7.01 \mathrm{~mL}, 87.1 \mathrm{mmol})$ for $16 \mathrm{~h}$, after which the solvent was removed in vacuo. To a solution of the crude formamide $(3.10 \mathrm{~g}, 15.5$ $\mathrm{mmol})$ in dry $\mathrm{CH}_{2} \mathrm{Cl}_{2}(45 \mathrm{~mL})$ was added $\mathrm{Et}_{3} \mathrm{~N}(4.32 \mathrm{~mL}, 31 \mathrm{mmol})$. The solution was cooled to $-40{ }^{\circ} \mathrm{C}$, after which triphosgene $(1.61 \mathrm{~g}$, $5.52 \mathrm{mmol}$ ) was added portionwise. The resulting mixture was slowly warmed to room temperature and stirred for $3 \mathrm{~h}$. Hereafter, the reaction was quenched with ice-water $(50 \mathrm{~mL})$, and the resulting biphasic system was separated. The organic layer was washed with 1 $\mathrm{M} \mathrm{HCl}$, saturated $\mathrm{NaHCO}_{3}$, and water, dried over $\mathrm{Na}_{2} \mathrm{SO}_{4}$, filtered, and concentrated in vacuo, affording the target isocyanide as a pale yellow oil $(2.471 \mathrm{~g}, 88 \%): R_{f}=0.16\left(\mathrm{cHex} / \mathrm{EtOAc} / \mathrm{Et}_{3} \mathrm{~N}\right.$ 4:1:0.01); ${ }^{1} \mathrm{H}$ NMR (500.23 MHz, $\mathrm{CDCl}_{3}$ ) $\delta 4.12(\mathrm{q}, J=7.0 \mathrm{~Hz}, 2 \mathrm{H}), 3.89-$ $3.81(\mathrm{~m}, 1 \mathrm{H}), 3.60(\mathrm{ddd}, J=3.5,8.0,14.0 \mathrm{~Hz}, 2 \mathrm{H}), 3.54-3.44(\mathrm{~m}$, $1 \mathrm{H}), 1.93-1.83(\mathrm{~m}, 2 \mathrm{H}), 1.83-1.75(\mathrm{~m}, 2 \mathrm{H}), 1.25(\mathrm{t}, J=7.0 \mathrm{~Hz}$, $3 \mathrm{H}) ;{ }^{13} \mathrm{C}\left(125.97 \mathrm{MHz}, \mathrm{CDCl}_{3}\right) \delta 156.2(\mathrm{t}, J=5.0 \mathrm{~Hz}), 155.2,61.6$, $49.3(\mathrm{t}, J=6.0 \mathrm{~Hz}), 40.0,31.2$, 14.6; HRMS (ESI) $\mathrm{m} / z$ calcd for $\mathrm{C}_{9} \mathrm{H}_{14} \mathrm{~N}_{2} \mathrm{O}_{2} \mathrm{Na}^{+}[\mathrm{M}+\mathrm{Na}]^{+}$205.0944, found 205.0955.

\section{ASSOCIATED CONTENT}

\section{S Supporting Information}

The Supporting Information is available free of charge on the ACS Publications website at DOI: 10.1021/acs.joc.7b02844.

${ }^{1} \mathrm{H}$ and ${ }^{13} \mathrm{C}$ NMR spectra for all new compounds (PDF)

\section{AUTHOR INFORMATION}

\section{Corresponding Authors}

*E-mail: r.v.a.orru@vu.nl.

*E-mail: e.ruijter@vu.nl

\section{ORCID 중}

Bert U.W. Maes: 0000-0003-0431-7606

Eelco Ruijter: 0000-0002-1105-3947

Notes

The authors declare no competing financial interest.

\section{ACKNOWLEDGMENTS}

We thank Elwin Janssen for NMR support and maintenance. The research leading to these findings has received financial support from The Netherlands Organization for Scientific Research (NWO).

\section{REFERENCES}

(1) Fidock, D. A. Nature 2010, 465, 297.

(2) Ridley, R. G. Nature 2002, 415, 686.

(3) Wellems, T. E. Nature 1992, 355, 108.

(4) Gregson, A.; Plowe, C. V. Pharmacol. Rev. 2005, 57, 117.

(5) Goulopoulou, S.; McCarthy, C. G.; Webb, R. C. Pharmacol. Rev. 2016, 68, 142.

(6) Madrid, P. B.; Sherrill, J.; Liou, A. P.; Weisman, J. L.; DeRisi, J. L.; Guy, R. K. Bioorg. Med. Chem. Lett. 2005, 15, 1015.

(7) Miller, L. H.; Ackerman, H. C.; Su, X.-z.; Wellems, T. E. Nat. Med. 2013, 19, 156.

(8) Gignoux, E.; Azman, A. S.; de Smet, M.; Azuma, P.; Massaquoi, M.; Job, D.; Tiffany, A.; Petrucci, R.; Sterk, E.; Potet, J.; Suzuki, M.; Kurth, A.; Cannas, A.; Bocquin, A.; Strecker, T.; Logue, C.; Pottage, T.; Yue, C.; Cabrol, J.-C.; Serafini, M.; Ciglenecki, I. N. Engl. J. Med. 2016, 374, 23.

(9) Madrid, P. B.; Chopra, S.; Manger, I. D.; Gilfillan, L.; Keepers, T. R.; Shurtleff, A. C.; Green, C. E.; Iyer, L. V.; Dilks, H. H.; Davey, R. A.; Kolokoltsov, A. A.; Carrion, R., Jr.; Patterson, J. L.; Bavari, S.; Panchal, R. G.; Warren, T. K.; Wells, J. B.; Moos, W. H.; Burke, R. L.; Tanga, M. J. PLoS One 2013, 8, e60579.

(10) Kužnik, A.; Benčina, M.; Švajger, U.; Jeras, M.; Rozman, B.; Jerala, R. J. Immunol. 2011, 186, 4794.

(11) Tewari, S.; Chauhan, P. M. S.; Bhaduri, A. P.; Fatima, N.; Chatterjee, R. K. Bioorg. Med. Chem. Lett. 2000, 10, 1409.

(12) Brouwer, C.; Jenko, K.; Zoghbi, S. S.; Innis, R. B.; Pike, V. W. J. Med. Chem. 2014, 57, 6240.

(13) Singh, S.; Roy, K. K.; Khan, S. R.; Kashyap, V. K.; Sharma, A.; Jaiswal, S.; Sharma, S. K.; Krishnan, M. Y.; Chaturvedi, V.; Lal, J.; Sinha, S.; Dasgupta, A.; Srivastava, R.; Saxena, A. K. Bioorg. Med. Chem. 2015, 23, 742.

(14) Shinkai, H.; Ito, T.; Iida, T.; Kitao, Y.; Yamada, H.; Uchida, I. J. Med. Chem. 2000, 43, 4667.

(15) Sestili, I.; Borioni, A.; Mustazza, C.; Rodomonte, A.; Turchetto, L.; Sbraccia, M.; Riitano, D.; Del Giudice, M. R. Eur. J. Med. Chem. 2004, 39, 1047.

(16) Kireev, D. B.; Chrétien, J. R.; Raevsky, O. A. Eur. J. Med. Chem. 1995, 30, 395.

(17) Oh, K. H.; Kim, J. G.; Park, J. K. Org. Lett. 2017, 19, 3994.

(18) Wezeman, T.; Zhong, S.; Nieger, M.; Bräse, S. Angew. Chem., Int. Ed. 2016, 55, 3823.

(19) Li, Y.; Zhang, L.; Zhang, L.; Wu, Y.; Gong, Y. Org. Biomol. Chem. 2013, 11, 7267. 
(20) Dhanapal, R.; Perumal, P. T.; Damodiran, M.; Ramprasath, C.; Mathivanan, N. Bioorg. Med. Chem. Lett. 2012, 22, 6494.

(21) Abbiati, G.; Arcadi, A.; Canevari, V.; Capezzuto, L.; Rossi, E. J. Org. Chem. 2005, 70, 6454.

(22) Vlaar, T.; Maes, B. U. W.; Ruijter, E.; Orru, R. V. A. Chem. Heterocycl. Compd. 2013, 49, 902.

(23) Tsai, J.-Y.; Chang, C.-S.; Huang, Y.-F.; Chen, H.-S.; Lin, S.-K.; Wong, F. F.; Huang, L.-J.; Kuo, S.-C. Tetrahedron 2008, 64, 11751.

(24) Ren, J.; Zhao, J.; Zhou, Y.-S.; Liu, X.-H.; Chen, X.; Hu, K. Med. Chem. Res. 2013, 22, 2855.

(25) Tollari, S.; Cenini, S.; Ragaini, F.; Cassar, L. J. Chem. Soc., Chem. Commun. 1994, 15, 1741.

(26) Li, L.; Wang, H.-K.; Kuo, S.-C.; Wu, T.-S.; Lednicer, D.; Lin, C. M.; Hamel, E.; Lee, K.-H. J. Med. Chem. 1994, 37, 1126.

(27) Sun, F.; Zhao, X.; Shi, D. Tetrahedron Lett. 2011, 52, 5633.

(28) Carta, D.; Bortolozzi, R.; Hamel, E.; Basso, G.; Moro, S.; Viola, G.; Ferlin, M. G. J. Med. Chem. 2015, 58, 7991.

(29) Strekowski, L.; Say, M.; Henary, M.; Ruiz, P.; Manzel, L.; Macfarlane, D. E.; Bojarski, A. J. J. Med. Chem. 2003, 46, 1242.

(30) Torii, S.; Okumoto, H.; Xu, L. H. Tetrahedron Lett. 1991, 32, 237.

(31) Torii, S.; Okumoto, H.; Xu, L. H.; Sadakane, M.; Shostakovsky, M. V.; Ponomaryov, A. B.; Kalinin, V. N. Tetrahedron 1993, 49, 6773.

(32) Åkerbladh, L.; Nordeman, P.; Wejdemar, M.; Odell, L. R.; Larhed, M. J. Org. Chem. 2015, 80, 1464.

(33) Tang, T.; Fei, X.-D.; Ge, Z.-Y.; Chen, Z.; Zhu, Y.-M.; Ji, S.-J. J. Org. Chem. 2013, 78, 3170.

(34) Kosugi, M.; Ogata, T.; Tamura, H.; Sano, H.; Migita, T. Chem. Lett. 1986, 15, 1197.

(35) Pan, Y.-Y.; Wu, Y.-N.; Chen, Z.-Z.; Hao, W.-J.; Li, G.; Tu, S.-J.; Jiang, B. J. Org. Chem. 2015, 80, 5764.

(36) Whitby, R. J.; Saluste, C. G.; Furber, M. Org. Biomol. Chem. 2004, 2, 1974.

(37) Vlaar, T.; Cioc, R. C.; Mampuys, P.; Maes, B. U. W.; Orru, R. V. A.; Ruijter, E. Angew. Chem., Int. Ed. 2012, 51, 13058.

(38) Craciunescu, D. G.; Doadrio, A.; Furlani, A.; Scarcia, V. Chem.Biol. Interact. 1982, 42, 153.

(39) Manzel, L.; Strekowski, L.; Ismail, F. M. D.; Smith, J. C.; Macfarlane, D. E. J. Pharmacol. Exp. Ther. 1999, 291, 1337.

(40) Strekowski, L.; Zegrocka, O.; Henary, M.; Say, M.; Mokrosz, M. J.; Kotecka, B. M.; Manzel, L.; Macfarlane, D. E. Bioorg. Med. Chem. Lett. 1999, 9, 1819.

(41) Herrera, L.; Stephens, D. E.; D’Avila, A.; George, K. G.; Arman, H.; Zhang, Y.; Perry, G.; Lleonart, R.; Larionov, O. V.; Fernandez, P. L. Org. Biomol. Chem. 2016, 14, 7053. 